\title{
Emittance, surface structure, and electron emission
}

\author{
Kevin L. Jensen ${ }^{*}$ \\ Code 6843, Naval Research Laboratory, Washington, DC 20375, USA \\ Donald A. Shiffler \\ Air Force Research Laboratory, Kirtland AFB, New Mexico 87117, USA \\ John J. Petillo \\ Leidos, Billerica, Massachusetts 01821, USA \\ Zhigang Pan \\ IREAP, University of Maryland, College Park, Maryland 20742, USA \\ John W. Luginsland \\ Air Force Office of Scientific Research, Arlington, Virginia 22203, USA
}

(Received 3 January 2014; published 15 April 2014)

\begin{abstract}
The emittance of high brightness electron sources, particularly field emitters and photocathodes but also thermionic sources, is increased by surface roughness on the emitter. Such structure causes local field enhancement and complicates both the prediction of emittance and the underlying emission models on which such predictions depend. In the present work, a method to find the emission trajectories near regions of high field enhancement is given and applied to emittance predictions for field, photo, and thermal emission for an analytically tractable hemispherical model. The dependence of the emittance on current density, spatial variation, and acceleration close to the emission site is identified and the impact of space charge discussed. The methodology is extensible to field emission from close-spaced wirelike structures, in particular, and extensions to that configuration are discussed. The models have application to electron sources for high frequency vacuum electronics, high power microwave devices, and free-electron lasers.

DOI: 10.1103/PhysRevSTAB.17.043402

PACS numbers: 29.25.Bx, 79.70.+q, 79.60.-i, 85.45.Db
\end{abstract}

\section{INTRODUCTION}

For accelerators and electron devices, the usefulness of a high brightness electron beam is constrained by its emittance [1], of which that portion due to the cathode, also known as "intrinsic emittance," is of increasing importance. Its quantification and prediction, particularly for field and photoemission sources, is complicated by emission nonuniformity and geometric effects. Applications for which the reduction of intrinsic emittance is paramount include, but are not limited to, high frequency vacuum electronics (VE) [2-5], high power microwave devices [6-8], free-electron lasers (FELs) [9-11], and electron beam lithography [12-14]. Particularly for field and photoemission, emittance is strongly coupled to space charge effects.

The present work analyzes a simple geometrical model to identify how roughness and field enhancement increases

\footnotetext{
*kevin.jensen@nrl.navy.mil

Published by the American Physical Society under the terms of the Creative Commons Attribution 3.0 License. Further distribution of this work must maintain attribution to the author $(s)$ and the published article's title, journal citation, and DOI.
}

emittance, and to vet methods designed for the future incorporation of space charge modifications. The organization of the present study is as follows. First, the importance of emittance is assessed. Second, a formulation of emittance valid near the cathode surface tailored to identifying the impact of geometry using a simple analytical model of a hemisphere is presented. Third, the impact of field enhancement on the initial (or "launch") velocities from which subsequent trajectories are determined is estimated. Fourth, numerical estimates of a geometrical array of emitters is considered, as is the manner in which the methodology may be extended to wirelike (carbon fiber) field emitters. Finally, a discussion of how the methodology may be modified to treat the consequences of space charge forces due to the emitted current, particularly with respect to beam optics codes, is given.

\section{EMITTANCE AND APPLICATIONS}

The role of emittance depends strongly on the physics of the device to which the beam is coupled. The challenges of linear beam devices exemplify present concerns.

Improvements in high power and high frequency vacuum electronics are associated with a reduction in 
physical dimensions [15,16]. Very high power devices require very large current densities, but thermionic cathodes are run $<1 \mathrm{~A} / \mathrm{cm}^{2}$ because their lifetime shortens [17]. Consequently convergence of the electron beam by factors of 100 or greater are necessitated to achieve high beam tunnel current densities if thermionic cathodes are used, motivating interest in nonthermionic sources, for which an understanding of the fundamental impact of emittance on the beam dynamics is essential [18]. The cross-sectional radius $R(z)$ of the beam is governed by the beam envelope equation $[1,19]$

$$
\frac{d^{2}}{d z^{2}} R+\left(\frac{q B}{2 \beta \gamma m c}\right)^{2} R-\frac{2 I_{a}}{(\gamma \beta)^{3} I_{o}} \frac{1}{R}-\frac{\varepsilon^{2}}{R^{3}}=0,
$$

where $B$ is the magnetic field, $I_{a}$ is the beam current, and $\varepsilon$ is the emittance. The factors in which they appear are referred to as the magnetic term, the space charge term, and the emittance term, respectively. The other terms are: $I_{o}=$ $q m c^{2} / \alpha \hbar=17.045 \mathrm{kA}$ is a characteristic current [20]; the relativistic factors $\beta=v / c$ and $\gamma=1 / \sqrt{1-\beta^{2}}$ are related to the beam acceleration potential $\varphi_{b} \equiv V_{b} / q$ by $\gamma m c^{2}=m c^{2}+V_{b}$, or

$$
(\beta \gamma)^{2}=\frac{V_{b}}{m c^{2}}\left(2+\frac{V_{b}}{m c^{2}}\right),
$$

so that $\beta \gamma \approx \sqrt{2 V_{b} / m c^{2}}$; if $f$ is the frequency, then $2 \pi f n R / c=$ a zero of the Bessel function, with $n$ an integer related to mode, entailing that frequency is inversely proportional to beam radius (higher order modes may be sought for higher frequency VE applications [15]); and lastly, the remaining factors have their usual interpretation as in Table I. Brillouin flow entails the sum of the magnetic, space charge, and emittance terms vanishing, and so the "smooth flow" condition is

$$
\frac{I_{a}}{\pi R^{2}}=\frac{I_{o}}{8 \pi}\left(\frac{2 V_{b}}{m c^{2}}\right)^{1 / 2}\left(\frac{q B}{m c}\right)^{2}\left\{1-\frac{8 m V_{b} \varepsilon^{2}}{q^{2} B^{2} R^{4}}\right\}
$$

which is expressed as

$$
J_{\text {beam }}(\varepsilon)=J_{\text {beam }}(0)\{1-\delta(\varepsilon)\} .
$$

Consequently, emittance reduces $J_{\text {beam }}$ by $\delta(\varepsilon)=$ $\left(8 m V_{b} / q^{2} B^{2}\right)\left(\varepsilon^{2} / R^{4}\right)$. Because technology constrains the magnetic field $B$ magnitude, and $V_{b}$ depends on the platform, the pursuit of higher frequency and therefore lower beam radius $R$ makes emittance reduction increasingly important as a consequence of $\delta \propto \varepsilon^{2} / R^{4}$, given that circuit interception by high current density beams is damaging to the small circuits of high power mmw-to$\mathrm{THz}$ VE devices [2].

For short wavelength [21,22] and high average power $[23,24]$ FELs, good gain requires the beam emittance to be smaller than the photon beam emittance $\lambda / 4 \pi$ [25]. The
TABLE I. Fundamental constants. Units are characteristic of emission phenomena: elementary charge $[q]$, length $[\mathrm{nm}]$, time $[\mathrm{fs}]$, energy $[\mathrm{eV}]$, and temperature $[\mathrm{K}]$ (for Kelvin).

\begin{tabular}{lccc}
\hline \hline Symbol & Definition & Value & Unit \\
\hline$q$ & Unit charge & 1 & $\mathrm{q}$ \\
$c$ & Speed of light & 299.792 & $\mathrm{~nm} / \mathrm{fs}$ \\
$m$ & Electron rest mass & 5.68563 & $\mathrm{eV} / c^{2}$ \\
$k_{B}$ & Boltzmann's constant & $(11604.5)^{-1}$ & $\mathrm{eV} / \mathrm{K}$ \\
$\hbar$ & Planck's constant & 0.658212 & $\mathrm{eV} / \mathrm{fs}$ \\
$\alpha$ & Fine structure constant & $1 / 137.036$ & $\cdots$ \\
$Q$ & $\alpha \hbar c / 4$ & 0.36 & $\mathrm{eV}-\mathrm{nm}$ \\
\hline \hline
\end{tabular}

importance of modeling the cathode emission physics and electron dynamics to understanding the interplay between emittance and space charge has therefore been advocated for cathode research and development [22], particularly in simulation codes [26]. The intrinsic emittance of the photocathodes are emerging as the primary limitation to realizing the short wavelengths and energy recovering linac sources of $\mathrm{x}$ rays and makes describing the causes of cathode emittance important $[22,27]$, with surface roughness emerging as a probable contributor [28-32].

Modeling emission and emittance at the cathode in particle-in-cell (PIC) simulation codes entails needs. First, a model of how emittance scales with feature geometry would be advantageous but is generally absent except for stylized geometries. Second, field emission structures can have dimensions and apex shapes that require a generalization of the point charge model (PCM) for conical emitters [33,34], such as the carbon fiber wires shown in Fig. 1. Third, space charge close to the emitters, be they conical or fiberlike, affects trajectories in a complex manner and necessitates a methodology that can account for it. The present work treats the first complication and does so in a manner that allows for the consideration of the second, deferring the third to a separate study. The present methodology has bearing on several emission mechanisms from spherical and conical as well as wirelike

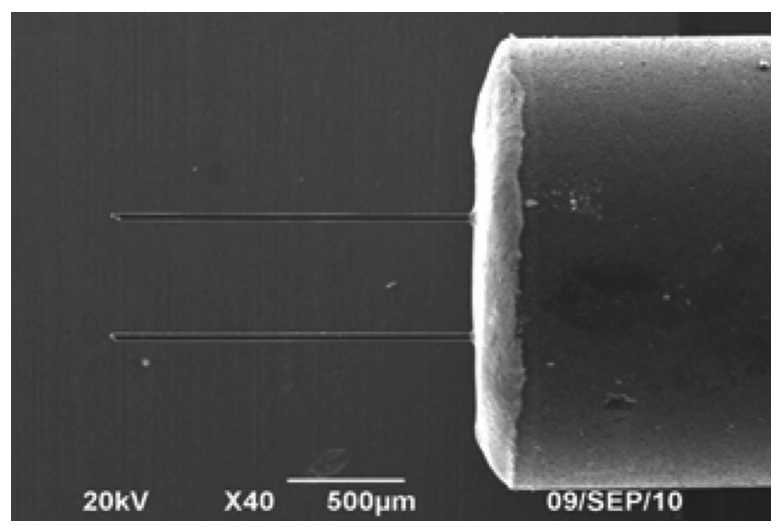

FIG. 1. Two single carbon fiber field emitters $35 \mu \mathrm{m}$ diameter and hundreds of $\mu \mathrm{m}$ in length. 
shapes, and therefore, the impact of surface structure on emittance for field emission and photoemission cathodes of VE and FELs can be addressed as well.

Apart from serving as an analytical model for emittance predictions, the hemispherical, or "boss," model that forms the basis of the present study is additionally used to test expectations arising from PIC simulations on the boss geometry that are intended to vet the trajectory and impulse models developed in Sec. IV. In particular, for boss parameters designed to evoke similar conditions of the wires of Fig. 1 (boss diameter of $35 \mu \mathrm{m}$ emitting on the order of a $\mu \mathrm{A}$ per wire with an apex field of roughly $3 \mathrm{GV} / \mathrm{m}$ and a work function of $4.9 \mathrm{eV}$ ), simulations using MICHELLE [26,35] for a boss on a plane conductor using a Fowler-Nordheim emission model [equivalent to Eq. (A9)] are shown in Figs. 2 and 3. The equipotential contour lines, field magnitudes, and trajectory shapes predicted by the analytical model must be comparable (and will be shown to be so). The PIC simulations reveal the importance of the space charge modification: although the emission currents from the wires result in a space-charge field reduction of less than $0.4 \%$, the resulting change in current is from $1.12 \mu \mathrm{A}$ to $1.04 \mu \mathrm{A}$, or a reduction of $7 \%$. A fuller account of the modeling of the wires will be taken up in a separate study, although a discussion of the connection to it will be given here.

Related prior treatments of surface roughness are as follows. Martinelli [36] considers an analytical 2D potential variation: his concern with the launch directions and the impact on the velocities is very similar to the approach here, particularly with regards to the "impulse approximation" discussed in Sec. IV B. Bradley et al. [28] theoretically

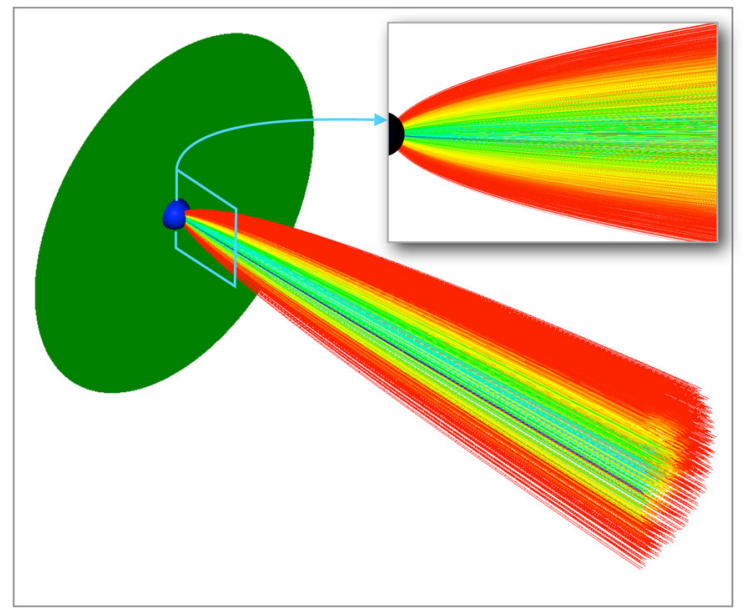

FIG. 2. Cross section of PIC simulation of field emission from hemispherical boss including space charge for $1 \mu \mathrm{A}$ currents. Apex field is $3 \mathrm{GV} / \mathrm{m}$, diameter is $35 \mu \mathrm{m}$. Space charge effects cause small field reductions of $<0.4 \%$ but have a strong impact on current $(-7 \%)$. Red trajectories have currents 2 orders of magnitude smaller than dark blue trajectories (visible from on-axis emission).

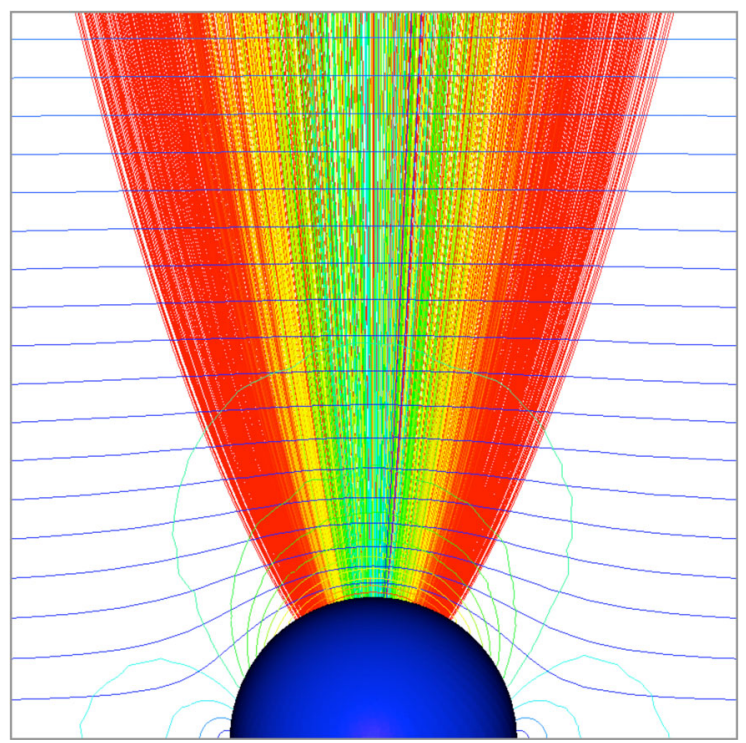

FIG. 3. Same as Fig. 2 inset but showing larger side view with overlaid equipotentials (thin dark blue lines) and field magnitudes (thin light blue lines). The "notional" emission angle introduced in Sec. III C and defined by Eq. (15) is comparable to the launch origin of the yellow lines.

examined the mean transverse emission energy of a negative electron affinity GaAs surface for which roughness was treated as a sinusoidal variation. They drew a distinction between a "field distortion effect" and a "slope effect," the former attributed to Martinelli and the later associated with electron emission normal to the surface. The normal launch is much like the "Ballistic approximation" considered in Sec. IV A. Graves et al. [37] observe that roughness increases the emittance of a copper photocathode by approximately a factor of 2. For GaAs photocathodes, Karkare and Bazarov [31] find that nanoscale roughness can increase emittance by almost an order of magnitude, and their simulations reveal that the inclusion of scattered electrons in the yield affects agreement with their experimental measurements. Krasilnikov [38] generalizes the 2D sinusoidal variation of a surface to 3D, as well as examines the Lorenztian model of Lau [39], to conclude that the increased electric field on the surface can lead to an increase in thermal emittance comparable to $30 \%$, and argues that space charge effects increase it; his conclusions are supported by the present work. He considers both "normal emission" (as done in the present analysis) in which the electron is launched perpendicular to the surface and the effects of an emission distribution rather than a perpendicular to the surface launch for, in particular, $\mathrm{Cs}_{2} \mathrm{Te}$ photocathodes for a rf photoinjector for the European XFEL.

A hemispherical representation of a protrusion, analogous to the hemispherical boss model here, was developed by Zhang, Lau, and Gilgenbach [40]. Qian et al. [32] consider a variation of the 2D sinusoidal surface roughness model (the analysis was expanded upon by Dowell et al. 
[41]). They argue that for photoemission, the planar emittance should be augmented by a roughness term according to $\varepsilon_{\text {total }}^{2}=\varepsilon_{\text {smooth }}^{2}+\varepsilon_{\text {rough }}^{2}$, where $\varepsilon_{\text {smooth }}$ is the planar (Dowell-Schmerge) formula of Eq. (C3) and $\varepsilon_{\text {rough }} \approx$ $\left(\rho_{c} / 2\right) \sqrt{X_{g} F / 3 m c^{2}}$ where $X_{g}$ is a geometrical factor due to surface roughness the sinusoidal model suggests is given by $X_{g} \approx 2 \pi^{2} a^{2} / 3 \lambda_{p}$ in the terms and units of the present work. The ratio $\left(\varepsilon_{\text {rough }} / \varepsilon_{\text {smooth }}\right)^{2} \approx X_{g} F /(\hbar \omega-\phi)=$ $45.2 \%$ for $F=50 \mathrm{eV} / \mu \mathrm{m}, a=100 \mathrm{~nm}, \lambda_{p}=10 \mu \mathrm{m}$, $\lambda=250 \mathrm{~nm}$, and $\Phi=4.5 \mathrm{eV}$ and $\phi=4.232 \mathrm{eV}$, numbers that are compatible with the present analysis.

The emittance of a single tip and an array of Spindt-type field emitters predicted by Jensen et al. $[42,43]$ relied on a combination of analytical (prolate spheroidal) geometries and the point charge model to follow electrons to an evaluation plane where the emittance was calculated by numerically finding the trajectories. The present work builds on those methods and endeavors to provide analytic models from which dependencies can be ascertained.

\section{EMITTANCE FOR A CURVED SURFACE}

\section{A. Moments and normalized emittance}

Emittance is a measure of the ability to focus a beam as well as its tendency to diverge as it propagates [44]. The rms emittance $\varepsilon_{x}$ is defined by [1]

$$
\varepsilon_{x}=\sqrt{\left\langle x^{2}\right\rangle\left\langle x^{\prime 2}\right\rangle-\left\langle x x^{\prime}\right\rangle^{2}}
$$

where the moments $\langle\ldots\rangle$ are evaluated with respect to a distribution of particles: if emission is cylindrically symmetric, then $\varepsilon_{x}=\varepsilon_{y}$, and this assumption shall be made. The factor $x^{\prime}$ is the ratio of an electron's transverse deviation $\Delta x$ after moving longitudinally forward $\Delta z$, or $x^{\prime}=\Delta x / \Delta z \approx v_{x} / v_{z}$, where $v_{x}=\hbar k_{x} / m$ and $v_{z}=\hbar k_{z} / m$ are the velocities in the $\hat{x}$ and $\hat{z}$ directions, respectively.

The emission distribution can be affected by how the cathode is employed such that emission is not a priori uniform (the beam can have density gradients at the edge), resulting in other distributions having greater fidelity. Examples of various distributions are KapchinskyVladimirsky (K-V), waterbag, parabolic, and Gaussian profiles, and their consideration will result in total emittances $\varepsilon_{\text {tot }}$ that are larger than $\varepsilon_{x}$, or $\varepsilon_{\text {tot }}=K \varepsilon_{x}$ where $K$ can range from $4(\mathrm{~K}-\mathrm{V})$ to $>10$ (Gaussian) [18,44]. Here, however, complexities associated with the distribution are subsidiary.

A relatively standard account is as follows. For beams that are rapidly accelerated at the cathode in high fields (of the order $\mathrm{MV} / \mathrm{m}$ for thermal emission, $20 \mathrm{MV} / \mathrm{m}$ for photoemission, and $>1 \mathrm{GV} / \mathrm{m}$ for field emission), $v_{z} \approx v \equiv \beta c$. Beams are accelerated rapidly so that $v_{z} \gg v_{x}, v_{y}$, or $\beta_{z} \approx \beta$, so that the "normalized" emittance $\varepsilon_{n, \mathrm{rms}}=\beta \gamma \varepsilon_{x}$ is given by [45]

$$
\varepsilon_{n, \mathrm{rms}} \equiv \frac{\hbar}{m c} \sqrt{\left\langle x^{2}\right\rangle\left\langle k_{x}^{2}\right\rangle-\left\langle x k_{x}\right\rangle^{2}}
$$

and is invariant for linear forces. For uniform and rotationally symmetric emission from a flat surface, $\left\langle x k_{x}\right\rangle$ vanishes, resulting in the emittance becoming a product of the rms radius of the cathode $\rho_{c}$ and the square root of the beam's energy spread: for thermionic emission, the energy of a gas of electrons is $3 k_{B} T / 2$, or $k_{B} T / 2$ in each orthogonal direction, so that for thermal emission from a uniform, flat, circular area [compare Eq. (C1)]

$$
\varepsilon_{n, \mathrm{rms}}=\frac{\rho_{c}}{2} \sqrt{\frac{k_{B} T}{m c^{2}}},
$$

a consequence of the Maxwell-Boltzmann distribution of emitted (thermal) electrons and the basis for referring to the "temperature" of an electron beam.

A similar expression is obtainable for photocathodes if the energy spread of the beam is taken as a reasonable approximation for the ratio of the transverse to longitudinal energy spread: Lee et al. [46] find, using $v_{x} / \beta \gamma c \approx(\beta \gamma)^{-1} \sqrt{2\left\langle E_{x}\right\rangle / m c^{2}}$, that for photocathodes, $\varepsilon_{n} \approx \rho_{c} \sqrt{2\left\langle E_{x}\right\rangle / m c^{2}}$. As with an ideal gas, if $\left\langle E_{x}\right\rangle=\langle E\rangle / 3 \approx(\hbar \omega-\phi) / 3$, then the equation of Lee et al. is similar to (but larger than) the more careful treatment of Dowell and Schmerge [47] used here and given by Eq. (C3).

Difficulties arise in this account near the surface of the cathode where $v_{z}$ is comparable to $v_{x}$ and a range of $v_{z}$ velocities exist. A curved surface entails that the field enhancement varies over the surface, so the acceleration the electrons experience depends on their emission location. It is therefore desirable to bring the electrons from the emission surface to a region (here termed the "virtual anode") where the usual emittance narrative is approximately valid. To do so, we shall begin with the simplest potential profile that allows an analytical solution, then demonstrate how to generalize it to treat emitters of far more relevant shapes.

\section{B. Potential and field for a hemisphere}

The potential associated with metallic hemispherical dome, or boss, is one of the simplest analytical models of a surface feature that an emitted electron may experience, and is defined in spherical coordinates $(r, \theta)$ by

$$
V(r, \theta)=V_{o}-F r \cos \theta\left[1-\left(\frac{a}{r}\right)^{3}\right]-\frac{2 Q a}{r^{2}-a^{2}},
$$

where $V_{o}=\mu+\Phi$ is the sum of the Fermi level $\mu$ and work function $\Phi, F$ is the product of the electric field in the $\hat{z}$ direction and the unit (electron) charge $q$ at the surface, and the term containing $Q=\alpha \hbar c / 4=0.36 \mathrm{eV}-\mathrm{nm}$ is the image charge for a sphere of radius $a$. On axis, with 
$r=a+z$, a Taylor expansion of the image charge term reveals $2 a Q /\left(r^{2}-a^{2}\right) \approx Q / z-Q /(2 a)+Q z /(2 a)^{2}$ to leading order; consequently, the usual image charge potential $V_{i}(z) \equiv \mu+\Phi-F z-Q / z$ can be retained with the replacements

$$
\begin{gathered}
\Phi \rightarrow \Phi_{a} \equiv \Phi+\frac{Q}{2 a}, \\
F \rightarrow F_{a} \equiv F-\frac{Q}{(2 a)^{2}} .
\end{gathered}
$$

The changes to the image charge potential are small: it is therefore useful to assume the radii-modified work function $\Phi_{a}$ and field $F_{a}$ are implicit in the current density equations (via the various emission models associated with thermal, field, and photoemission [48]) from which the emission probability factors shall be evaluated below. With that assumption in mind, the " $a$ " subscript will be suppressed.

The radial gradient of the potential evaluated at the surface tracks the variation in field down the side of the boss and is evaluated from Eq. (8) without the image charge term, or

$$
F(r, \theta)=-\partial_{r} V(r, \theta)=F_{o} \cos \theta\left[1+2\left(\frac{a}{r}\right)^{3}\right],
$$

where $F_{o}$ is taken to be the asymptotic, or far, field. A peculiar feature of the boss potential is that the field enhancement along the surface, $F(a, \theta)=3 F_{o} \cos \theta$, is independent of the radius of the boss, in contrast to conical and ellipsoidal emitters for which the field enhancement varies as the inverse radius of the apex of the emitter.

Importantly, the radial field component approaches its asymptotic form of $F_{o} \cos \theta$ as $(a / r)^{3}$, so that when the electron reaches $r \rightarrow 2 a$ the field guiding the electron's path is within $25 \%$ of its asymptotic value, that is, the field enhancement near the boss operates only for a short time before the electron experiences a constant field $F_{o}$ in the $\hat{z}$ direction. Therefore, the physics of the electron's trajectory may be accounted for by assessing the impulse contribution to the electron's initial velocity $v_{o}$, and subsequently following the trajectory in a constant field $F \rightarrow F_{o} \hat{z}$, a trivial problem in kinematics resulting in the coordinates for trajectory being

$$
\begin{gathered}
x(t)=a \sin \theta \cos \varphi+v_{o} t \sin \theta \cos \varphi, \\
z(t)=a \cos \theta+v_{o} t \cos \theta+\frac{F_{o}}{2 m} t^{2},
\end{gathered}
$$

from which the velocities are easily found $[y(t)$ is neglected for brevity], from which it follows

$$
x^{\prime}(t)=\frac{v_{x}(t)}{v_{z}(t)}=\frac{v_{o} \sin \theta}{v_{o} \cos \theta+(F t / m)} \cos \varphi .
$$

The moments of Eq. (5) may now be assessed.

\section{Notional and emission parameters}

When comparing the thermal, photo, and field emission mechanisms, using scales characteristic of each process allows for their direct comparison, a theme that will reappear in Sec. V. A natural scale is given by Eq. (14). Therefore, distances shall be scaled compared to $a$ and time to $\tau \equiv m v_{o} / F_{o}$ such that $t=p \tau$, in terms of which $\beta_{z}=(p+1) v_{o} / c$. This will entail forming estimates of the launch velocity $v_{o}$.

Electrons will not be uniformly emitted down the side of the boss if the probability of emission $P$ depends on surface field $P[F(\theta)] \equiv P(\theta)$. To develop a relation that relates the variation of emittance to changes in the underlying parameters, usage of an emission angle that captures the variation is required. Here, the concept of "notional emission area" from field emission studies [49] is used: by defining the total current from the boss as a product of the apex current density and an area factor, the angle describing the area factor gives a measure of the active, or emitting, region of the boss, even though the notional area is smaller than the actual or active area. Thus, the "notional" angle $\theta_{o}$ will allow scaling relations to be established, although a more refined estimate of emittance requires knowledge of the actual change of emission across the boss using an emission probability approach.

The notional emission angle $\theta_{o}$ is defined by equating the area of a uniformly emitting cap characterized by $\theta_{o}$ to the actual or integrated emission:

$$
\int_{0}^{\theta_{o}} 2 \pi a^{2} \sin \theta d \theta=\int_{0}^{\pi / 2} 2 \pi a^{2} P(\theta) \sin \theta d \theta
$$

where $P(\theta)$ is the probability of emission and is given by the ratio of the current density at $\theta$ compared to the apex value, or $P(\theta) \equiv J[F \cos (\theta)] / J[F]$ where $F$ is the apex field and $J$ is an emission equation [Eq. (A2)]. The related factor $\eta \equiv \cos \theta_{o}$ is notationally more convenient than working with $\theta_{o}$. The product of the notional emission area with the apex current density results in the total current from a protrusion. Each emission process depends on field differently, and so the related notional emission angle parameter $\eta \equiv \cos \left(\theta_{o}\right)$ will differ for thermal, photo, and field emission.

The initial, or launch, velocity $v_{o}$ of the electrons will be taken as the average velocity $\langle v\rangle$ obtained from $J=q \rho\langle v\rangle$ where

$$
v_{o}=\langle v\rangle \equiv \frac{\int_{0}^{\infty}(\hbar k / m) D(k) f(k) d k}{\int_{0}^{\infty} D(k) f(k) d k}
$$


in which $D(k)$ is the transmission probability and $f(k)$ is the supply function in a one-dimensional treatment leading to, for example, Eq. (A2). An analogous equation holds for $\left\langle v^{2}\right\rangle$, needed to evaluate the standard deviation, and is obtained by the replacement $\hbar k / m \rightarrow(\hbar k / m)^{2}$ in the numerator of Eq. (16), such that the rms velocity is $v_{\mathrm{rms}}=\sqrt{\left\langle v^{2}\right\rangle}=(\hbar / m) \sqrt{\left\langle k^{2}\right\rangle}$. The proportionality between $v_{o}$ and $\langle v\rangle$ is developed in Sec. IV for a curved surface; for a flat surface, they would be equal.

From the definition of $P(\theta)$, it is seen that Eq. (15) is the ratio of a total current $I_{\text {boss }}$ with its apex current density $J(F)$ : the integrand therefore indicates how much of the emitted current comes from each differential ribbon of area $2 \pi a^{2} \sin \theta d \theta$, which, when integrated, gives the current emitted within the polar angle $\theta$, or

$$
\Delta(\theta) \equiv \frac{I(\theta)}{I_{\mathrm{boss}}}=\frac{1}{1-\eta} \int_{0}^{\theta} \sin \theta^{\prime} P\left(\theta^{\prime}\right) d \theta^{\prime}
$$

for which $\Delta(\theta) \rightarrow 1$ as $\theta \rightarrow \pi / 2$, and is shown, along with $d \Delta / d \theta$, for all three processes in Figs. 4 and 5.

Expressions for $\eta, \Delta(\theta),\langle v\rangle$ and $v_{\text {rms }}$ are now developed for the thermal, field, and photoemission processes using the emission equations for $J_{\mathrm{FN}}, J_{\mathrm{RLD}}$, and $Q E_{\mathrm{FD}}$ given by Eqs. (A9), (A6), and (A12), respectively, for parameters based on Table III (see Sec. V for more discussion of parameters chosen).

\section{Thermal emission}

The field dependence resides in the Schottky factor reduction of the work function $\phi=\Phi-\sqrt{4 Q F}$. Using Eq. (A6) and letting $b$ represent the characteristic energy ratio, then

$$
P_{T}(\theta)=\exp [b(\sqrt{\cos \theta}-1)]
$$

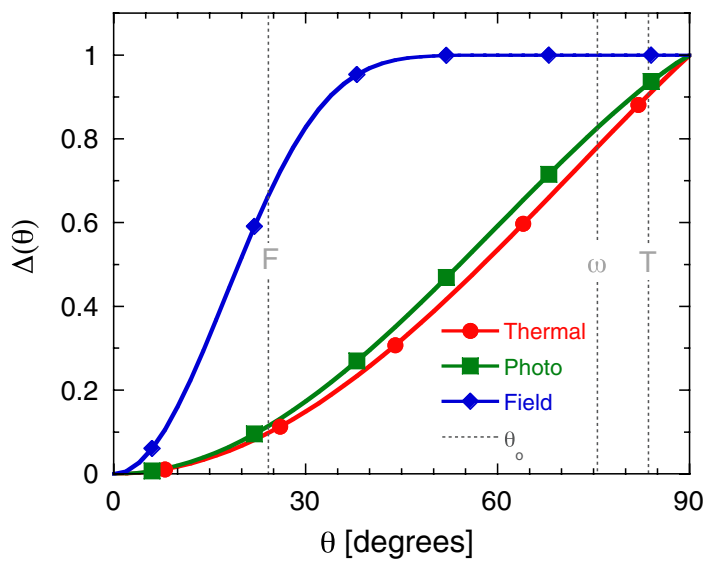

FIG. 4. Integrated current ratio $\Delta(\theta)$ [Eqs. (21), (27), (33)] evaluated for parameters of Table III. Dashed gray vertical lines correspond to $\theta_{o}$ for each process, labeled "T," " $\omega$," and "F," for circle (red line), square (green line), and diamond (blue line), respectively.

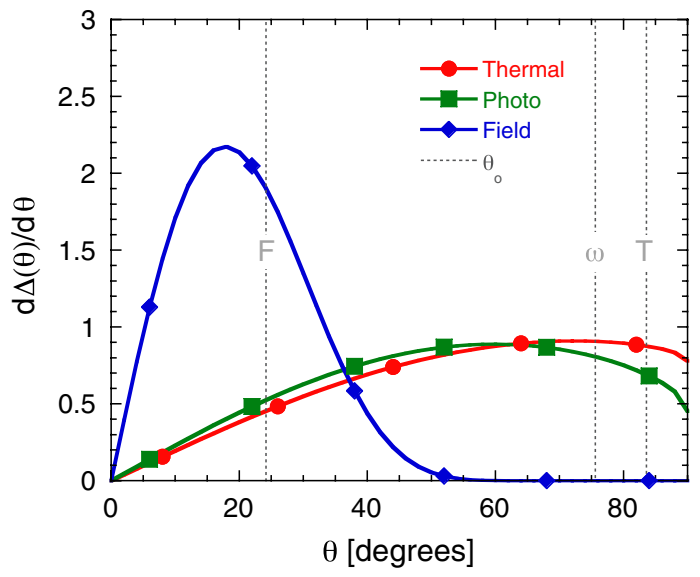

FIG. 5. Same as Fig. 4 but for $d \Delta / d \theta$.

$$
b=\beta \sqrt{4 Q F},
$$

resulting in

$$
\eta_{t}=1-\frac{2}{b}+\frac{2}{b^{2}}\left(1-e^{-b}\right)
$$

The integrated current fraction is

$$
\Delta_{T}(\theta)=1-\frac{1+(b \sqrt{\cos \theta}-1) e^{b \sqrt{\cos \theta}}}{1+(b-1) e^{b}} .
$$

The launch velocity and rms velocity are given by

$$
\begin{gathered}
v_{o}=\langle v\rangle=\left(2 k_{B} T / \pi m\right)^{1 / 2}, \\
v_{\text {rms }}=\sqrt{\left\langle v^{2}\right\rangle}=\left(k_{B} T / m\right)^{1 / 2} .
\end{gathered}
$$

\section{Field emission}

The field dependence is explicit in the current density. Using Eq. (A9), then

$$
\begin{gathered}
P_{f}(\theta)=(\cos \theta)^{2-\nu} \exp \left(b-\frac{b}{\cos \theta}\right), \\
b=B \Phi^{3 / 2} / F,
\end{gathered}
$$

resulting in, to a good approximation,

$$
\eta_{f}=\frac{b+3-\nu}{b+4-\nu}
$$

The integrated current fraction is, to a good approximation

$$
\Delta_{F}(\theta)=1-\exp \left\{-(b+4-\nu)\left(\frac{1}{\cos \theta}-1\right)\right\} \text {. }
$$


The launch velocity and rms velocity are approximately given by

$$
\begin{gathered}
v_{o}=\langle v\rangle=\frac{\hbar k_{F}}{m}\left(1-\frac{\Phi}{3 t_{o} b \mu}\right)^{2}, \\
v_{\mathrm{rms}}=\sqrt{\left\langle v^{2}\right\rangle}=\frac{\hbar k_{F}}{m}\left(\frac{3 t_{o} b \mu-\Phi}{3 t_{o} b \mu+\Phi}\right)^{2},
\end{gathered}
$$

where $\hbar k_{F}$ is recognized as the Fermi momentum.

\section{Photoemission}

The field dependence resides in the Schottky factor reduction of the work function $\phi=\Phi-\sqrt{4 Q F}$. Using Eq. (A12), then

$$
\begin{gathered}
P_{\omega}(\theta)=b^{-2}(b-1+\sqrt{\cos \theta})^{2}, \\
b=\frac{\hbar \omega-\phi}{\sqrt{4 Q F}},
\end{gathered}
$$

resulting in

$$
\eta_{\omega}=\frac{4 b-1}{6 b^{2}} .
$$

The integrated current fraction is

$\Delta_{\omega}(\theta)=1-\cos \theta \frac{6(b-1)^{2}+8(b-1) \sqrt{\cos \theta}+3 \cos \theta}{6(b-1)^{2}+8(b-1)+3}$.

Evaluation of the launch and rms velocities is complicated by the change in energy as the electron passes from bulk to vacuum. As is commonly done (e.g., Ref. [47]), the difference between the vacuum level and the conduction band minimum, being the height of the barrier $\mu+\phi$, is removed from the energy of the emitted electrons: the velocities are calculated with the prescription of $E^{\prime}=E+\hbar \omega-(\mu+\phi)$. Another complication is that the photon energy $\hbar \omega$ appears in addition to the energies represented in $b$ : consequently, it is convenient to perform expansions in terms of the small quantity $y b(\Phi / \mu)=(\hbar \omega-\phi) / \mu$ and it is found that on the vacuum (primed) side

$$
\begin{gathered}
v_{o}^{\prime}=\langle v\rangle^{\prime}=\frac{\hbar k_{F}}{m}\left[\frac{\hbar \omega-\phi}{3 \mu}\left(1-\frac{\hbar \omega-\phi}{8 \mu}\right)\right]^{1 / 2}, \\
v_{\mathrm{rms}}^{\prime}=\frac{\hbar k_{F}}{m}\left[\frac{\hbar \omega-\phi}{3 \mu}\left(1-\frac{\hbar \omega-\phi}{12 \mu}\right)\right]^{1 / 2} .
\end{gathered}
$$

The values of the initial velocity $\langle v\rangle$, notional angle factor $\eta$, and $b$ factors are shown for typical values in
Table III; here and below, the more convenient notation $v_{o}$ will be used in place of $\langle v\rangle$.

\section{Single boss emittance}

For a quantity $O$ defined on the surface of the boss, the evaluation of $\langle O\rangle$ is obtained by

$$
\langle O\rangle \approx \frac{1}{4 \pi} \int_{0}^{\theta_{o}}\left(\int_{0}^{2 \pi} O(\theta, \varphi) d \varphi\right) \sin \theta d \theta,
$$

where $\theta$ is the polar angle and $\varphi$ the azimuthal angle. From the forms of $x(t)$ and $x^{\prime}(t)$ in Eqs. (12) and (14), we find

$$
\begin{gathered}
\left\langle x(t)^{2}\right\rangle=\frac{1}{12}(2+\eta)(1-\eta)^{2}\left(p \tau v_{o}\right)^{2}, \\
\left\langle x^{\prime}(t)^{2}\right\rangle=\frac{1}{4} S(\eta), \\
\left\langle x(t) x^{\prime}(t)\right\rangle=\frac{1}{4} p \tau v_{o} R(\eta),
\end{gathered}
$$

where

$$
S(x)=\left(\frac{p+x}{p+1}\right)(2 p+x-1)-2 p \ln \left(\frac{p+x}{p+1}\right),
$$

$R(x)=\frac{1}{2}\left[(p-x)^{2}-(p-1)^{2}\right]+\left(p^{2}-1\right) \ln \left(\frac{p+x}{p+1}\right)$.

For the nonrelativistic case $(\gamma \approx 1)$ the emittance is then

$$
\varepsilon_{x}=\frac{1}{4 \sqrt{3}} \beta \tau p v_{o} \sqrt{(1-\eta)^{2}(2+\eta)^{2} S(\eta)-3 R(\eta)^{2}} .
$$

Although Eq. (42) is analytic, it is not computationally useful: identifying the asymptotic (large $p$ ) behavior is difficult and, more importantly, calculations are problematic because both terms in the radical are separately very large but close in value, introducing a possibility for roundoff error. A formulation that clearly gives the asymptotic behavior is as follows. Introduce $f$ and $g$ such that

$$
\begin{gathered}
\left\langle x^{2}\right\rangle=\frac{1}{4} N\left(v_{o} p \tau\right)^{2} \int_{0}^{\theta_{o}} g(\theta) d \theta, \\
\left\langle x^{\prime 2}\right\rangle=\frac{N}{4 p^{2}}\left(v_{o} p \tau\right)^{2} \int_{0}^{\theta_{o}} f(\theta)^{2} g(\theta) d \theta, \\
\left\langle x x^{\prime}\right\rangle=\frac{1}{4} N v_{o} p \tau \int_{0}^{\theta_{o}} f(\theta) g(\theta) d \theta,
\end{gathered}
$$

where $f(\theta)=p /(p+\cos \theta), g(\theta)=(\sin \theta)^{3} / N$, and $N=$ $(1-\eta)^{2}(2+\eta) / 3$ such that $f \leq 1$ and $g$ is normalized. It follows $\left\langle f^{2}\right\rangle-\langle f\rangle^{2}=\langle f-\langle f\rangle\rangle^{2}$. Define $\langle f\rangle \equiv f_{o}$. Then 


$$
\begin{gathered}
\varepsilon_{x}^{2}=\left(\frac{N v_{o} \tau}{4}\right)^{2} \int_{0}^{\theta_{o}}\left(f(\theta)-f_{o}\right)^{2} g(\theta) d \theta, \\
f_{o}=\int_{0}^{\theta_{o}} f(\theta) g(\theta) d \theta .
\end{gathered}
$$

An approximation of $f_{o}$ makes use of a Taylor expansion of $f(\theta)$ about $\cos \theta=s \approx 1$ : each term in the expansion can be analytically integrated given the form of $g(\theta)$. Retention of only the first term in the expansion yields the approximation

$$
1-f_{o}=\frac{p}{N} \int_{\eta}^{1} \frac{1-s^{2}}{p+s} d s \approx \frac{3(1+\eta)^{2}}{4(p+1)(2+\eta)} .
$$

Employing the same approach in the consideration of Eq. (46) results in

$$
\begin{aligned}
\left\langle\left(f-f_{o}\right)^{2}\right\rangle & =\frac{f_{o}^{2}}{N} \int_{\eta}^{1} \frac{\left(1-s^{2}\right)\left(s-s_{a}\right)^{2}}{(p+s)^{2}} d s \\
& \approx \frac{f_{o}^{2}(1-\eta)^{2}\left(3 \eta^{2}+18 \eta+19\right)}{80(p+1)^{2}(2+\eta)^{2}},
\end{aligned}
$$

where

$$
s_{a}=p\left(\frac{1-f_{o}}{f_{o}}\right) \approx \frac{3}{4}\left(\frac{1+\eta}{2+\eta}\right)^{2}
$$

marks the location (other than $s=1$ ) where the integrand vanishes. Assembling the components gives the leading approximation to $\varepsilon_{n, \mathrm{rms}}$ as

$$
\begin{aligned}
\varepsilon_{n, \mathrm{rms}} & =\frac{1}{4} \beta \tau v_{o} N \sqrt{\int_{0}^{\theta_{o}}\left(f(\theta)-f_{o}\right)^{2} g(\theta) d \theta} \\
& \approx C_{o} f_{o}(1-\eta)^{3} \sqrt{3 \eta^{2}+18 \eta+19},
\end{aligned}
$$

where

$$
C_{o} \equiv \frac{\sqrt{5}}{240}\left(\frac{\tau v_{o}^{2}}{c}\right)
$$

Observe that $f_{o} \rightarrow 1$ as $p \rightarrow \infty$, which is important, as it shows that $\varepsilon_{n, \text { rms }}$ is asymptotically independent of $p$ and therefore time.

The form of Eq. (53) allows three observations to be immediately made. First, because $\tau=m v_{o} / F$, the emittance scales as $v_{o}^{3} / F$. Second, because the emittance varies as $(1-\eta)^{3}$ and $\eta$ is near unity for field emission, field emission sources have small emittance which decreases rapidly as the field decreases, and as a consequence, field emission sources are very bright. Third, for emission from a single boss emitter, the emittance is independent of the boss

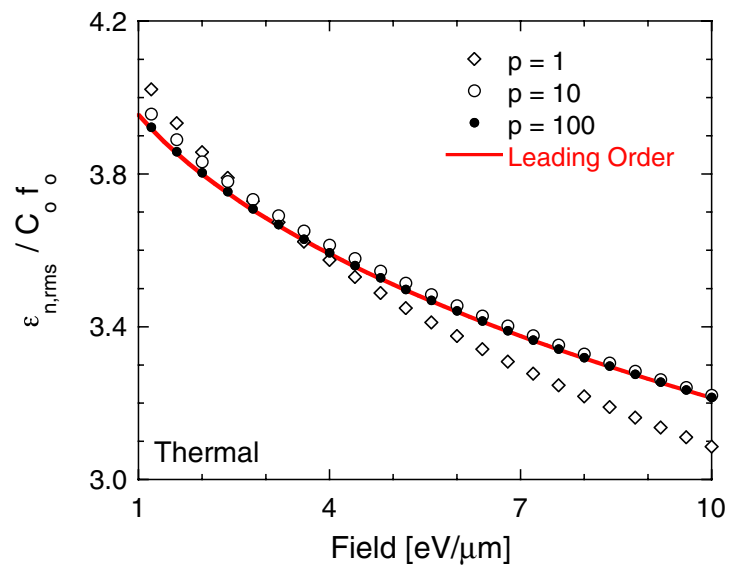

FIG. 6. Behavior of the ratio $\varepsilon_{n, \text { rms }}$ as calculated using Eq. (52) with $C_{o} f_{o}$ as calculated using Eq. (54) for parameters typical of thermal emission in Table III. The (red) solid line labeled "Leading Order" refers to the ratio of Eq. (53) with Eq. (54), or $\left[(1-\eta)^{3} \sqrt{3 \eta^{2}+18 \eta+19}\right]$.

radius-rather, the length scale is set by $m c^{2} / F$ - and dependent on the product of two factors: the first is a coefficient $C_{o} \propto v_{o}^{3} / F$ and the second is a factor dependent only on $\eta$. The behavior of the latter is indicated in Figs. 6, 7, and 8 for thermal, photo, and field emission parameters, respectively, in which the ratio $\varepsilon_{n, \mathrm{rms}} / C_{o} f_{o}$, calculated directly from Eq. (52), is shown as a function of $F$ for fields representative of each technology; although Eq. (52) is well approximated by Eq. (53) (as shown in Fig. 9 for representative fields of each emission mechanism), the red line also shown is the ratio of Eq. (53) with $C_{o} f_{o}$ to show the approach to the asymptotic limit. The representative fields are $[F]_{T}=0.1 \mathrm{eV} / \mu \mathrm{m},[F]_{\omega}=10 \mathrm{eV} / \mu \mathrm{m}$, and $[F]_{F}=1 \mathrm{eV} / \mathrm{nm}$, for thermal, photo, and field emission, respectively, as indicated by the subscript: the use of representative fields allows the $x$ axis in the figures to be standardized, as in $F=\chi[F]_{x}$ for " $x$ " representing the emission mechanism and $\chi$ ranging from 1 to 10 .

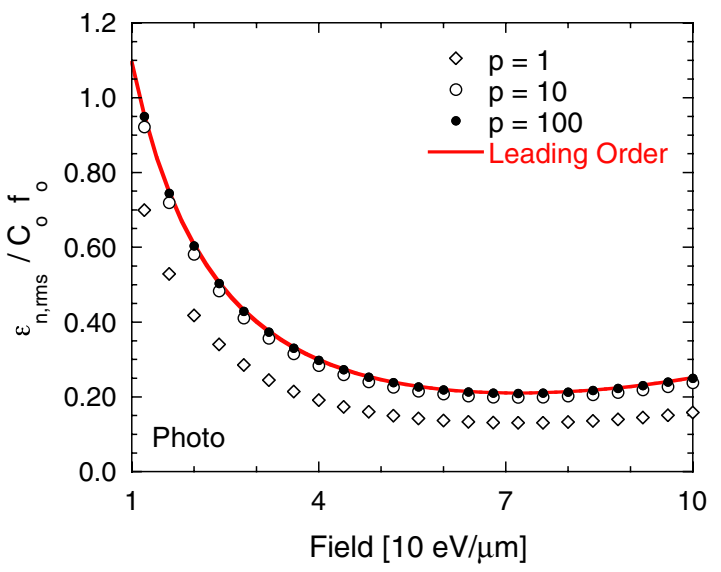

FIG. 7. Same as Fig. 6 but for parameters typical of photoemission in Table III. 


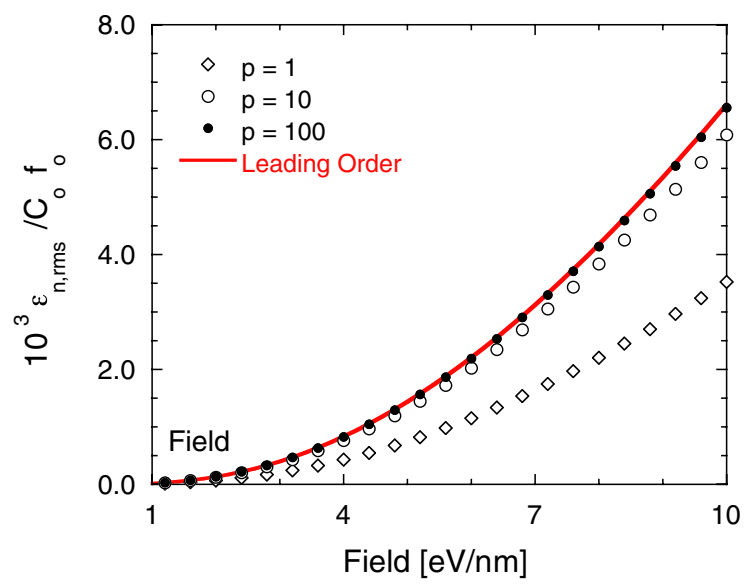

FIG. 8. Same as Fig. 6 but for parameters typical of field emission in Table III.

In terms of $\chi=F /[F]_{x}$, the magnitude of $C_{o}$ for each emission mechanism is considered for energies (e.g., $k_{B} T$, $\mu$, and $\Phi$ ) measured in $[\mathrm{eV}]$, and using the launch velocity $v_{o}$ for each process, it is found

$$
\begin{gathered}
C_{o}[T]=6.62 \times 10^{-5} \frac{\left(k_{B} T\right)^{3 / 2}}{\chi}, \\
C_{o}[\omega]=7.095 \times 10^{-5} \frac{(\hbar \omega-\Phi)^{3 / 2}}{\chi}, \\
C_{o}[F] \approx 3.686 \times 10^{-8} \frac{\mu^{3 / 2}}{\chi},
\end{gathered}
$$

where $C_{o}$ is measured in [mm-mrad] and, for field emission $[F]$, only the leading order term is shown, being $~ 100$ times larger than the next order term. These three equations

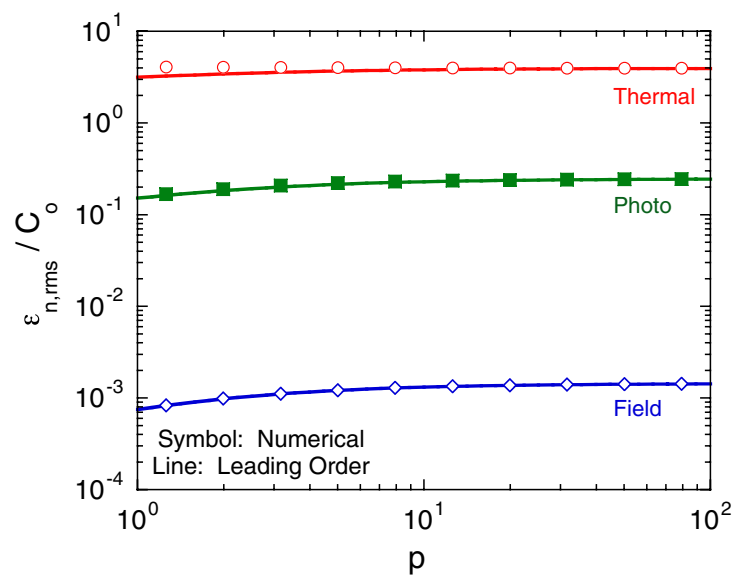

FIG. 9. Behavior of the ratio $\varepsilon_{n, \mathrm{rms}}$ as calculated using Eq. (52) with $C_{o}$ as a function of the time parameter $p=t / \tau$ for the representative fields $[F]_{x}$ of each mechanism $(x=T, F, \omega)$. The (red) solid line labeled "Leading Order" refers to the ratio of Eq. (53) with $C_{o}$ of Eq. (54). coupled with Figs. 6, 7, and 8, specify the single boss emittance for the $T, F$, and $\omega$ mechanisms.

\section{E. Array of bosses emittance}

Although realistic thermionic and photoemission surfaces have surface features varying in size and randomly placed, and field emitters can be made in triangular arrays rather than on a square lattice, for the analytic model, assume that first, the $M^{2}$ protrusions are uniformly sized; second, that the protrusions are located on a square array with a pitch of $l$ (distance from boss to boss) so that the area of the array is $[(M-1) l]^{2} \equiv L^{2}$; and third, that $l$ is sufficiently large that to a good approximation, the trajectories of a single boss are unaffected by its neighbors. The consequences of not making the third approximation will be the subject of a separate study.

The $x$ location of the bosses $X_{i j}$ is obtained by appending the location of the center to $x(t)$ and so

$$
\begin{gathered}
x(t) \rightarrow X_{i j}(t)=x_{i j}+x(t), \\
x_{i j}=(2 i-M-1) l,
\end{gathered}
$$

such that $(M-1) l=L / 2$ and the manner in which $\langle\ldots\rangle$ is evaluated is now taken to be

$$
\left\langle X_{i j}(t)^{2}\right\rangle=\frac{1}{4 \pi M^{2}} \sum_{i=1}^{M} \sum_{j=1}^{M} \int X_{i j}(t)^{2} d \Omega
$$

where the $d \Omega$ integration is as in Eq. (15). Finally, assume that $L^{2} \gg\left\langle x(t)^{2}\right\rangle$, leading to the independence of $X_{i j}$ on $j$. As a consequence of $\left\langle x_{i j}\right\rangle=0$ we have

$$
\begin{aligned}
\left\langle X_{i j}(t)^{2}\right\rangle & =\left\langle x_{i j}^{2}\right\rangle+\left\langle x(t)^{2}\right\rangle \\
& =\frac{1}{3}\left(M^{2}-1\right) l^{2}+\left\langle x(t)^{2}\right\rangle,
\end{aligned}
$$

where $\left(M^{2}-1\right) l^{2} / 3=(M+1) L^{2} / 12(M-1) \approx L^{2} / 12$, a result anticipated from similar field emitter array evaluations [33]. The first term dominates, and therefore $\left\langle X_{i j}(t)^{2}\right\rangle$ is independent of $p$ to leading order and is of the size $L^{2} / 12$. As with the single boss emittance, it is seen that the array emittance is the product of a coefficient $L v_{o} / 12 c$ and a factor only dependent on $\eta$.

In the case of thermionic emission, the form of $\varepsilon_{\text {array }}$ harkens to Eq. (7). Insofar as $\rho_{c} \approx L / \sqrt{\pi}$ and $v_{o}=\sqrt{2 / \pi} v_{\mathrm{rms}}$, then

$$
\frac{\varepsilon_{\text {array }}^{T}}{\varepsilon_{1 D}^{T}}=\frac{1-\eta}{6} \sqrt{2+\eta} \sqrt{2},
$$

where $\varepsilon_{1 D}^{T}$ is given by Eq. (C1). The ratio is approximately 0.32 over the range of fields characteristic of thermionic 
emission. This finding emphasizes an important distinction: intrinsic emittance in the presence of surface roughness has two components, the first due to the well-known transverse velocity spread in the emission process itself which gives rise to $\varepsilon_{\text {therm }}$, and the second due to surface curvature and the launching of electrons normal to a curved surface giving rise to $\varepsilon_{\text {array }}$. Curvature therefore increases emittance as total emittance is the square root of the sum of the squares. The effect will be magnified below, where $v_{o}$ shall be altered to account for surface fields providing acceleration near the boss apart from the background field.

In the case of field emission, for which the flat surface emittance is given by Eq. (C2), and simplifying by neglecting small terms, it is found

$$
\frac{\varepsilon_{\text {array }}^{F}}{\varepsilon_{1 D}^{F}} \approx \frac{1-\eta}{6} \sqrt{2+\eta}\left(\frac{3 \pi t_{o} b \mu}{4 \Phi}\right)^{1 / 2},
$$

where $b$ is given in Eq. (25). For the field emission parameters of Table III, the ratio is 0.1408. Observe, however, that unlike either thermal emission or photoemission, the emission will appear as an array of emission sites smaller in size than either the hemispherical bosses or their pitch, which may allow for advantageous alterations of the emittance phase space [50], in addition to the other opportunities that field emission cathodes present [51] that are precluded for both thermionic and photoemission.

In the case of photoemission, for which the flat surface emittance is given by Eq. (C3), it is seen that

$$
\frac{\varepsilon_{\text {array }}^{\omega}}{\varepsilon_{1 D}^{\omega}}=\frac{1-\eta}{6} \sqrt{2+\eta}\left[2 \pi \frac{\hbar \omega-\Phi}{\hbar \omega-\phi}\right]^{1 / 2} \text {. }
$$

For the photoemission parameters of Table III, the ratio is 0.3384 . For a cesiated surface $(\Phi=1.8 \mathrm{eV})$ in the green $(\lambda=532 \mathrm{~nm})$, the ratio becomes 0.3638 . Curvature therefore contributes to the larger experimentally measured emittance found by Dowell et al. [47] to be roughly a factor of 2 larger than the theoretical value, other causes of the increase being space charge forces [52] and sources of nonuniform emission. For multialkali antimonide photocathodes, simple roughness models have been used with success to model emittance data [53] and have been invoked in explaining experimentally larger emittance values measured after cleaning processes thought to increase surface roughness for copper [32].

It is seen that compared to the $1 \mathrm{D}$ emittance equations, each process is the product of a factor $(1-\eta) \sqrt{2+\eta} / 6$ (smallest for field emission because $\eta$ is close to unity) with a process-dependent term varying between $\sqrt{2}$ (thermal) and $>5$ (field). For Table III parameters, the values of $10^{4} \times\left(\varepsilon_{1 D} / \rho_{c}\right)$ are $2.25,8.34$, and 2.14 for thermal, field, and photoemission, respectively. The final contribution that affects the emittance, considered next, is the impulse contribution to the launch velocity.

\section{SURFACE LAUNCH VELOCITIES}

\section{A. Integration of trajectories}

The "ballistic" trajectories entailed by Eq. (12) presume that the field is constant and in the $\hat{z}$ direction; the field on the surface of the boss given by Eq. (11) shows that a field in the $\hat{\rho}$ direction $F_{\rho}$ is strong and present, and that $F_{z}$ near the apex is $3 \times$ background, as shown in Figs. 10 and 11, compared to the surface field, as the radial distance $r$ increases, $F(r, \theta) / F(a, \theta)=1+2(a / r)^{3}$, so that the field has declined to within $10 \%$ of background by $r=$ $\sqrt{\rho^{2}+z^{2}}=2.7$ as suggested by the flattening of the gray equipotential lines indicated in Figs. 12, 13 and 14. The effects of fields at the surface of the boss can therefore be approximated by providing an impulse to the initial, or launch, velocity of the electron and making the replacement $v_{o} \rightarrow \sigma v_{o}$ in the calculation of emittance.

The method to approximate $\sigma$ is best determined rewriting the ballistic equations of Eq. (12), but work with $\rho(t)^{2}=x(t)^{2}+y(t)^{2}$, so that the positions are calculated according to a variant of Simpson's rule given by

$$
\vec{r}(t+\delta t)=\vec{r}(t)+\frac{a \delta t}{2 v_{o} \tau}[\vec{v}(t+\delta t)+\vec{v}(t)],
$$

where $\vec{r}(t)=[\rho(t), z(t)]$ and where the velocities are updated according to

$$
\vec{v}(t+\delta t)=\vec{v}(t)+\frac{\delta t}{m} \vec{F}[\rho(t), z(t)]
$$

Observe that Eqs. (66) and (67) are standard methods for finding trajectories for small $\delta t$ when $\vec{F}(\rho, z)$ varies.

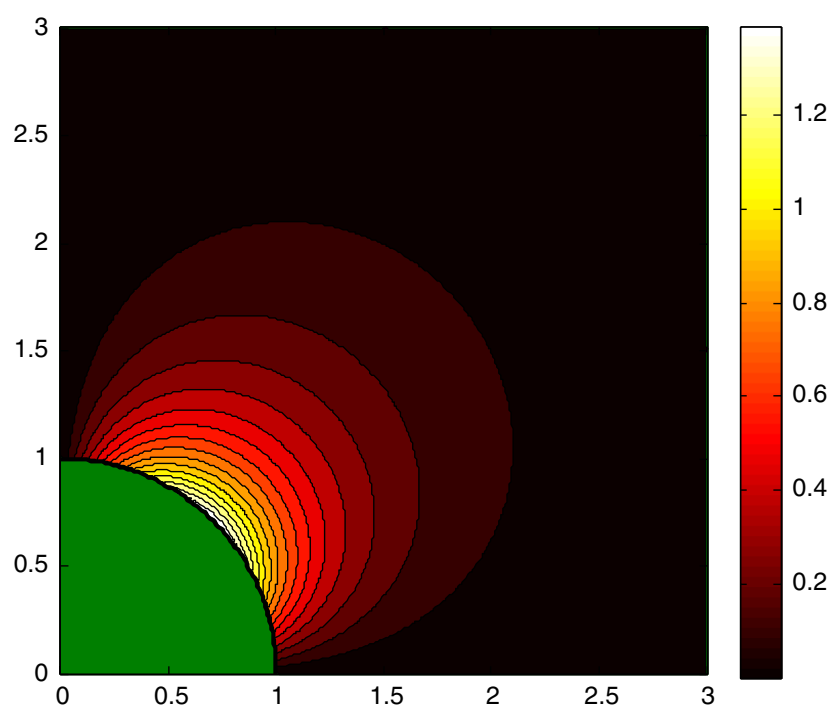

FIG. 10. Transverse field $F_{\rho}$ for a boss normalized with respect to the background field $F_{o}$. On the surface of the boss, $F_{\rho} / F_{o}=3 \sin \theta \cos \theta$. The boss is the green hemisphere in the lower left. 


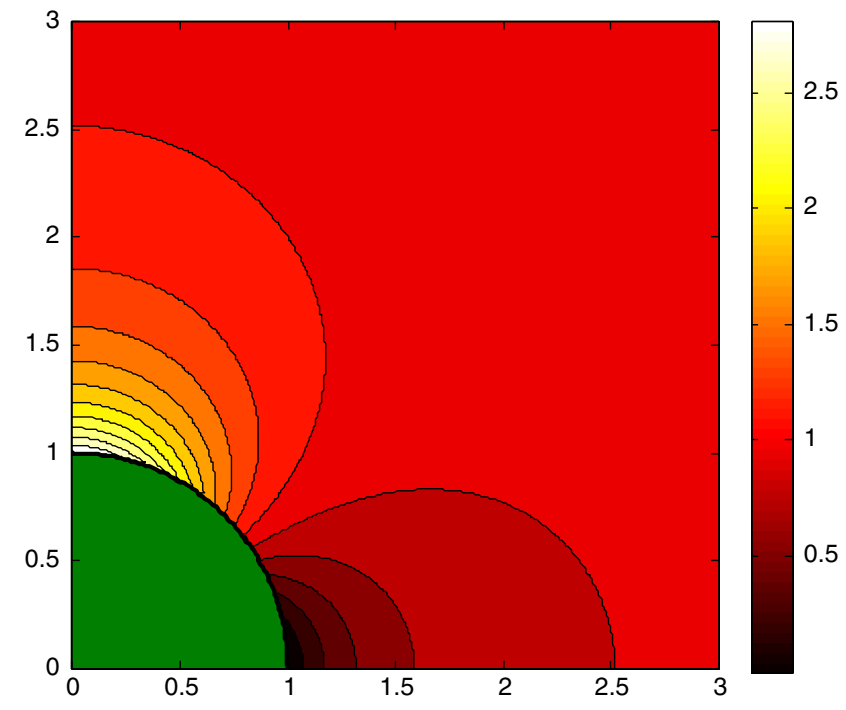

FIG. 11. Longitudinal field $F_{z}$ for a boss normalized with respect to the background field $F_{o}$. On the surface of the boss, $F_{z} / F_{o}=3 \cos ^{2} \theta$. The boss is the green hemisphere in the lower left.

\section{B. Impulse approximation}

In the case of constant forces, the approximation behind Eqs. (66) and (67) are exact, allowing a useful representation of the ballistic approximation to be obtained by replacing $t \rightarrow 0$ and $t+\delta t \rightarrow p\left(a / v_{o}\right) \equiv p \delta$; when coupled with the initial positions and velocities on the surface of the boss and $\vec{F} \rightarrow F_{o} \hat{z}$ we find

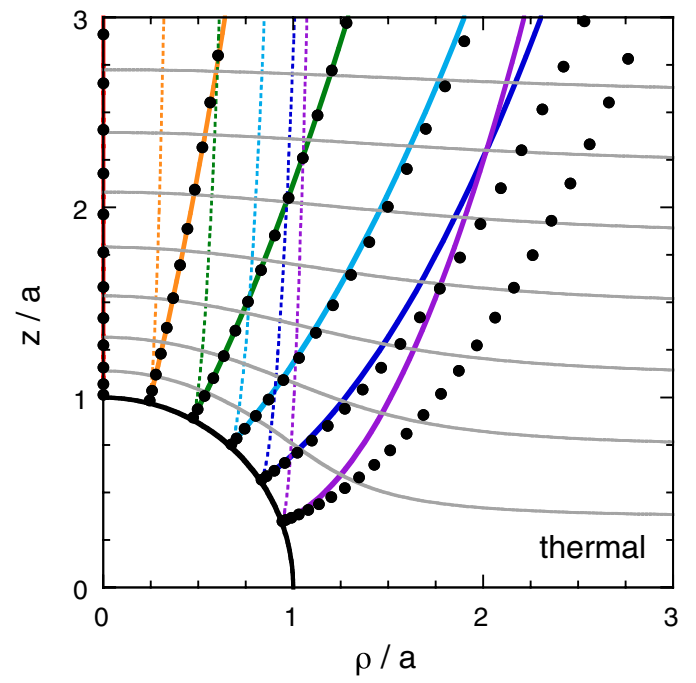

FIG. 12. Trajectories associated with thermal emission from a hemispherical boss without (dashed lines) and with (solid lines) the impulse approximation, compared to numerically evaluated trajectories (solid dots) for thermal emission using Table III parameters. The (purple) last line is launched from the notional angle $\theta_{o}$. Gray lines correspond to equipotentials equally spaced in energy.

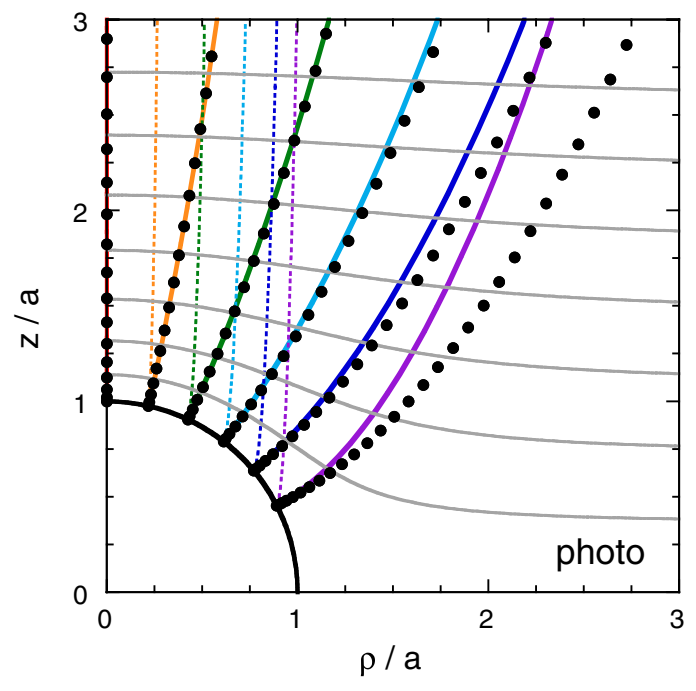

FIG. 13. Same as Fig. 12 but for photoemission.

$$
\frac{\rho(p \delta)}{a}=(1+p \cos \theta) \sin \theta
$$

$$
\frac{z(p \delta)}{a}=(1+p \cos \theta) \cos \theta+\frac{1}{2} \kappa p^{2}
$$

where

$$
\kappa \equiv \frac{F_{o} a}{m v_{o}^{2}}=\frac{F_{o} \delta}{m v_{o}}
$$

is a ratio of the energy scale associated with the field and that associated with the initial kinetic energy. The "impulse approximation" is then tantamount to finding $\sigma$ such that

$$
\frac{1}{2} m v_{o}^{2}\left(\sigma^{2}-1\right)=V(0, a)-V(0, n a)
$$

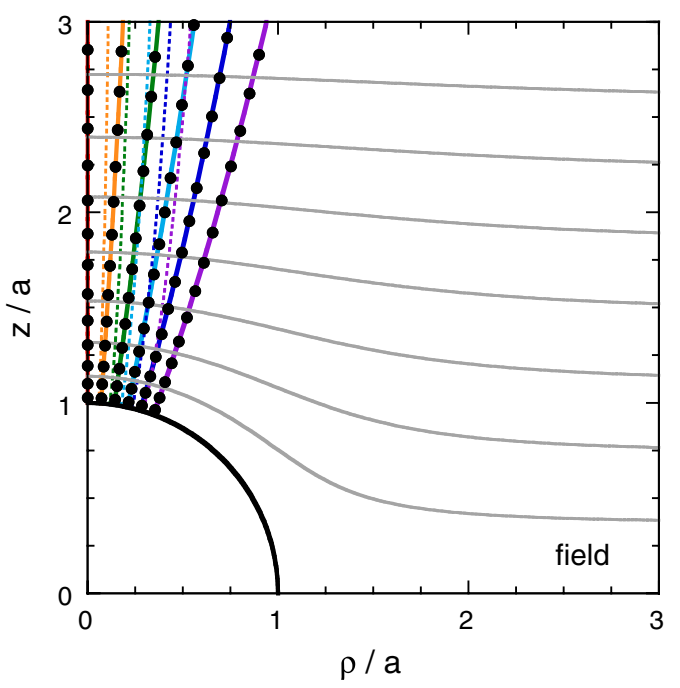

FIG. 14. Same as Fig. 12 but for field emission. 
where $V(x, a)$ is the potential energy, and $z=n a$ is sufficiently far above the boss apex that the potential is reasonably flat there. We find

$$
\sigma^{2}=1+\frac{2 \kappa}{n^{2}}\left(n^{3}-1\right)
$$

Taking $n=2$ as suggested by Figs. 13 and 14 gives $\sigma^{2}=1+\frac{7}{2} \kappa$. Including the impulse approximation into the ballistic equations then amounts to making the replacement

$$
1+p \cos \theta \rightarrow 1+\sigma p \cos \theta
$$

in Eqs. (68) and (69) to account for the increased launch velocity associated with a curved surface. The procedure is general: although applied to a boss here for analytical convenience, the method can be extended to equipotentials associated with more complex geometries, as shall be the subject of a separate study. The values of $\eta, \sigma$, and $\kappa$ for the emission mechanisms are shown in Table III for typical parameters using the aforementioned approximations.

\section{Performance of impulse approximation}

The impulse approximation assumes that the trajectories are given by the ballistic equations of Eqs. (68) and (69) where the initial velocities are augmented as prescribed by Eq. (73). The performance of this approximation is compared to solving the equations of motion numerically through an iterated use of Eqs. (66) and (67) for small values of $\delta t$ for thermal (Fig. 12), photo (Fig. 13), and field emission (Fig. 14) conditions with parameters as specified by Table III.

In each figure, the ballistic equations without (dashed lines) and with (solid lines) the impulse approximation are compared to the numerically calculated trajectories (solid black dots) such that the last trajectory (purple line with the largest initial $\theta$ ) is launched from $\theta=\theta_{o}$ for each process. Clearly, the impulse approximation significantly corrects the error that would otherwise be associated with the ballistic approach. The fields associated with field emission are so large that seeing the differences on the same scales of the thermal and photo trajectories is difficult: a longer view is shown in Fig. 15 but is such that the vertical and horizontal axes are using different scales which also causes the hemispherical boss to appear flattened. In all cases the impulse approximation performs reasonably well for trajectories whose launch $\theta<60^{\circ}$, which is all the trajectories for field emission, but approximately half the current for thermal and photoemission, as suggested by Fig. 4. Moreover, the findings compare well with the PIC simulations of Figs. 2 and 3.

Because the outer impulse approximation lines have smaller transverse velocities $\left(v_{\rho}\right)$ than the numerically evaluated trajectories, it is seen that the impulse

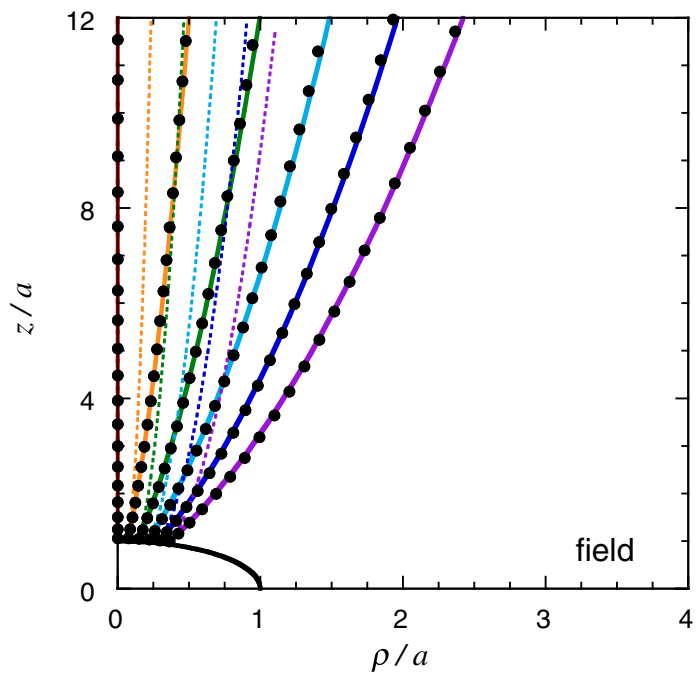

FIG. 15. Same as Fig. 14 but with the axes adjusted to see trajectories well away from the boss structure.

approximation serves as a lower limit to the estimation of emittance from a hemispherical structure. Therefore, Eq. (63) allows for the conclusion

$$
\varepsilon \gtrsim \varepsilon_{\text {array }} \equiv \sqrt{2+\eta}(1-\eta) \frac{L \sigma v_{o}}{12 c} .
$$

\section{NUMERICAL RESULTS}

\section{A. Characteristic array emittances}

Emission mechanisms are varied and so therefore will be the parameters associated with them, making comparisons challenging. Comparisons will consequently be based on characteristic operating conditions for representative configurations of each technology for an array of hemispherical bosses. Three quantities shall be evaluated for each case using a representative value of $L$ and the remaining parameters as given in Tables II and III: (i) the numerical value of the array emittance $\varepsilon_{\text {array }}$ evaluated according to Eq. (74), (ii) a comparison of $\varepsilon_{\text {array }}$ the corresponding 1D equations of Appendix C, and lastly, (iii) a comparison of $\varepsilon_{\text {array }}$ to a reference flat thermionic emitter $0.2 \mathrm{~cm}$ in radius operated at $T=1273$ Kelvin as evaluated by Eq. (C1), for which the emittance is approximately $0.463 \mathrm{~mm}$-mrad.

\section{Thermal emission}

For thermionic emission characteristic of dispenser cathodes used in high frequency microwave amplifiers, an extraction grid at hundreds of volts is positioned a fraction of a millimeter above the cathode surface. If a space charge limited current [see Eq. (B1)] of $J_{\mathrm{CL}}=$ $2 \mathrm{~A} / \mathrm{cm}^{2}$ for an anode-cathode gap of $D=250 \mu \mathrm{m}$, then $V_{a} / D=0.2638 \mathrm{eV} / \mu \mathrm{m}$ [54]. A background field of $F_{o}=$ $0.333 \mathrm{eV} / \mu \mathrm{m}$ is therefore away from the space charge limit but comparable to extraction grid technology. If the cathode 
TABLE II. Emission parameters. Terms on which current density depends. $R_{y}$ is the Rydberg energy and characteristic of atomic scales.

\begin{tabular}{lccc}
\hline \hline Symbol & Definition & Relation & Unit \\
\hline$k_{F}$ & Fermi wave number & $\ldots$ & $1 / \mathrm{nm}$ \\
$\mu$ & Chemical potential & $\hbar^{2} k_{F}^{2} / 2 m$ & $\mathrm{eV}$ \\
$\rho_{o}$ & Number density & $k_{F}^{3} / 3 \pi^{2}$ & $\# / \mathrm{cm}^{3}$ \\
$V$ & $q \times$ potential & $q \varphi$ & $\mathrm{eV}$ \\
$F$ & $q \times$ E field & $q \mathcal{E}$ & $\mathrm{eV} / \mathrm{nm}$ \\
$\beta$ & Inverse temperature & $1 / k_{B} T$ & $\mathrm{eV}-1$ \\
$\Phi$ & Work function & $4.5(\mathrm{Cu})$ & $\mathrm{eV}$ \\
$\phi$ & Schottky lowered $\Phi$ & $\Phi-\sqrt{4 Q F}$ & $\mathrm{eV}$ \\
$v_{o}$ & Launch velocity & $\mathrm{Eq} .(16)$ & $\cdots$ \\
$\tau$ & Characteristic time & $m v_{o} / F$ & $\cdots$ \\
$a$ & Boss radius & $\cdots$ & $\mathrm{nm}$ \\
$\eta$ & Notional cosine & $\mathrm{cos}\left(\theta_{o}\right)$ & $\cdots$ \\
$R_{y}$ & $(1 / 2) m(\alpha c)^{2}$ & 13.606 & $\mathrm{eV}$ \\
$A$ & $q / 16 \pi^{2} \hbar$ & $1.5414 \times 10^{-6}$ & $\mathrm{Amp} / \mathrm{eV}^{1}$ \\
$B$ & $4 \sqrt{2 m} / 3 \hbar$ & 6.8309 & $1 / \mathrm{nm}-\mathrm{eV}{ }^{1 / 2}$ \\
$A_{\mathrm{RLD}}$ & $2 q m\left(k_{B} / 2 \pi \hbar\right)^{2}$ & 120.17 & $\mathrm{Amp} / \mathrm{K}^{2} \mathrm{~cm}^{2}$ \\
$\zeta(2)$ & Riemann $\zeta$ function & $\pi^{2} / 12$ & $\cdots$ \\
\hline \hline
\end{tabular}

radius is $0.2 \mathrm{~cm}$, then the current is $250 \mathrm{~mA}$. A square of size $L=0.3536 \mathrm{~cm}$ has the same area. Machining of the cathode surface produces feature sizes comparable to a few $\mu \mathrm{m}$ and larger [55].

For a thermionic emitter $0.3536 \mathrm{~cm}$ on a side, $\varepsilon_{\text {array }}=17.0 \mathrm{~mm}$-mrad, which is 21.34 times larger than the $1 \mathrm{D}$ emittance, and 36.6 times larger than the reference emittance.

TABLE III. Parameters and values. Representative metallic parameters (nominally based on copper). Numbers in the "Units" column reference equations in the text. A dash "..." denotes term not needed for $\eta, \sigma, \kappa$, or $\Delta$.

\begin{tabular}{lcccc}
\hline \hline Term & Unit & Thermal & Field & Photo \\
\hline$a$ & $\mathrm{~nm}$ & 2000 & 5 & 200 \\
$F$ & $\mathrm{eV} / \mathrm{nm}$ & 0.001 & 8 & 0.075 \\
$T$ & $\mathrm{~K}$ & 1200 & $\ldots$ & $\ldots$ \\
$\mu$ & $\mathrm{eV}$ & $\ldots$ & 7 & 7 \\
$\Phi$ & $\mathrm{eV}$ & $\ldots$ & 4.5 & 4.5 \\
$\lambda$ & $\mathrm{nm}$ & $\ldots$ & $\ldots$ & 250 \\
$\nu$ & $\mathrm{A} 10$ & $\ldots$ & 0.77282 & $\ldots$ \\
$b$ & $19,25,31$ & 0.36696 & 8.1509 & 2.3978 \\
$\eta$ & $20,26,32$ & 0.1119 & 0.9121 & 0.2491 \\
$\theta_{o}$ & $\mathrm{arccos}(\eta)$ & $83.6^{\circ}$ & $24.2^{\circ}$ & $75.6^{\circ}$ \\
$\phi$ & $\mathrm{eV}$ & $\ldots$ & 1.1059 & 4.1714 \\
$\hbar k_{F} / m$ & $\mathrm{~nm} / \mathrm{fs}$ & $\ldots$ & 1.569 & 1.569 \\
$v_{o}=\langle v\rangle$ & $\mathrm{nm} / \mathrm{fs}$ & 0.1076 & 1.492 & 0.3018 \\
$\tau$ & $\mathrm{fs}$ & 18590 & 3.350 & 1657 \\
$\sigma$ & 72 & 10.36 & 3.472 & 15.95 \\
$\kappa$ & 70 & 30.38 & 3.159 & 72.40 \\
$\Delta\left(\theta_{o}\right)$ & $21,27,33$ & $90.5 \%$ & $66.6 \%$ & $82.6 \%$ \\
\hline \hline
\end{tabular}

\section{Field emission}

A field emitter array used for a $100 \mathrm{~W}$ traveling-wave tube produced a current of $100 \mathrm{~mA}$ from a cathode diameter of $1 \mathrm{~mm}$ using 50,000 emitters spaced $4 \mu \mathrm{m}$ apart [16]. If more than $40 \%$ of the emitters are responsible for all of the current from tips $5 \mathrm{~nm}$ in apex radius, then the apex fields are comparable to $F=8 \mathrm{eV} / \mathrm{nm}$, a third of which is $F_{o}=2.67 \mathrm{eV} / \mathrm{nm}$. A square of size $L=886 \mu \mathrm{m}$ has the same area.

For an array of field emitter protrusions $886 \mu \mathrm{m}$ on a side, $\varepsilon_{\text {array }}=2.30 \mathrm{~mm}$-mrad, which is 3.11 times larger than the 1D emittance, and 1.60 times larger than the reference emittance.

\section{Photoemission}

The first operation of a photoinjector at high average power [56] used a multialkali antimonide photocathode illuminated by a $527 \mathrm{~nm}$ laser in a field of $26 \mathrm{MV} / \mathrm{m}$, suggesting $F_{o}=26 \mathrm{eV} / \mu \mathrm{m}$, and the laser spot size on the cathode was 3-5 mm FWHM at that time. Such fields are similar to surface fields in more recent photoinjectors for x-ray FELs of $24 \mathrm{MV} / \mathrm{m}$ [57]. Contemporary studies of metal photocathodes using a $1 \mathrm{~mm}$ diameter beam suggest surface roughness on the order of a $\mu \mathrm{m}$ [58] [59]. A square of size $L=886 \mu \mathrm{m}$ has a comparable area.

For a photoemitter $886 \mu \mathrm{m}$ on a side, $\varepsilon_{\text {array }}=$ $16.0 \mathrm{~mm}$-mrad, which is 50.4 times larger than the $1 \mathrm{D}$ emittance, and 0.685 times smaller than the reference emittance.

\section{B. Variations on characteristic conditions}

As a consequence of the dependences implicit in the notional angles, the imparted impulses, and the launch velocities, the array emittance will change as conditions on the cathode change. The dependence of the various processes on their respective emission mechanisms (e.g., field for field emission) can be compared as follows.

The reference emittances will be taken as those found above, namely, $17.0 \mathrm{~mm}$-mrad (thermal), $2.30 \mathrm{~mm}$-mrad (field), and $16.0 \mathrm{~mm}$-mrad (photo). Changes to those reference values are considered as a function of the variation of its characteristic parameter $x$ where $x$ is the parameter with relation to a reference value. For thermal emission, $x=T /(1200 \mathrm{~K})$ and $x=1$ is the reference case. For field emission, $x=F /(6 \mathrm{eV} / \mu \mathrm{m})$, and $x=1.33$ is the reference case. For photoemission, $x=\lambda /(180 \mathrm{~nm})$, and $x=1.38$ is the reference case. Because the reference cases were typical cases, the scale factors for field emission and photoemission $(6 \mathrm{eV} / \mu \mathrm{m}$ and $180 \mathrm{~nm}$, respectively) were adjusted so that the intended range of $x$ values would not become unphysical or result in disallowed values. With the chosen scalings, values of $x=0.5,0.75,1.0,1.25$, and 1.50 were considered to show the variations to be expected in $\varepsilon_{\text {array }}$; the results are shown in Fig. 16. 


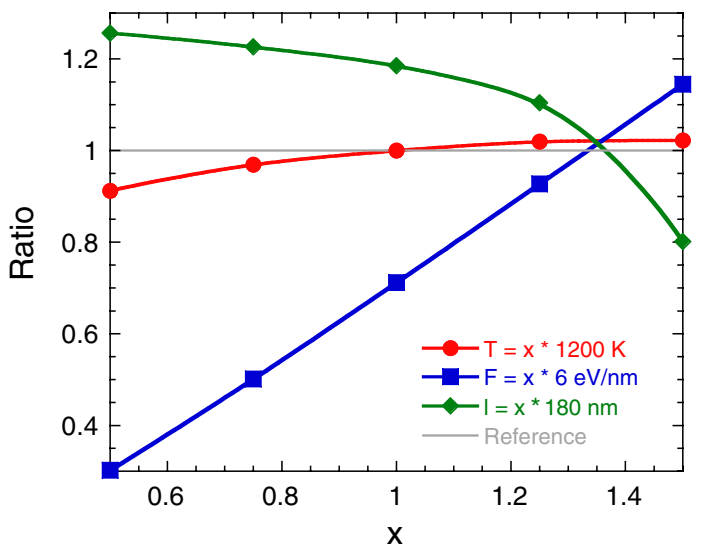

FIG. 16. Ratio of array emittances with reference cases $\left(\varepsilon_{\text {ref }}^{T}=\right.$ $\left.17.0 \mathrm{~mm}-\mathrm{mrad} ; \quad \varepsilon_{\text {ref }}^{F}=2.30 \mathrm{~mm}-\mathrm{mrad} ; \quad \varepsilon_{\mathrm{ref}}^{\omega}=16.0 \mathrm{~mm}-\mathrm{mrad}\right)$ as a function of the ratio of the independent parameter with $T_{\text {scale }}=1200 \mathrm{~K}$ (thermal), $F_{\text {scale }}=6 \mathrm{eV} / \mathrm{nm}$ (field), and $\lambda_{\text {scale }}=$ $180 \mathrm{~nm}$ (photo).

\section{Extension to wire field emitters}

The methodology developed above (though applied to hemispherical bosses because emittance due to surface roughness was the focus) can be extended to treat the carbon fiber wires shown in Fig. 1, as the techniques used are not beholden to the form of Eq. (8) for the potential. From PIC simulations of a hemispherical cap atop a tall cylindrical emitter of dimensions comparable to the carbon fiber wires, the total current increases by a factor of 4 times even as the current density at the apex is the same: more current is drawn from the sides of the hemispherical cap because the field falloff is slower. More can now be said about requirements on PIC and beam optics codes that may otherwise have difficulty in resolving length scales associated with realistic emission sites.

Consider simulations of field emission from a model of the fibers of Fig. 1 using the particle-in-cell code MICHELLE [26,35] shown in Fig. 18. By comparison to the hemisphere on plane of the surface roughness model, parallel wire emitters will introduce two significant modifications that increase the emittance, and can be reasonably well understood by attaching a cylindrical base to the hemispherical cap, as seen in Fig. 17. First, the equipotential lines are pulled down alongside the cylinder, causing the notional angle $\theta_{o}$ to increase. If $\theta_{o}$ for the hemispherical cap on post geometry of Fig. 17 has increased from $24^{\circ}$ to $45^{\circ}$, then (as simulated) the total emitted current will increase by a factor of 4 , and the emittance, as per Eq. (53) (assuming $C_{o} f_{o}$ is unchanged, although it, too, shall increase), will increase by a factor of 36.4 times for the same on axis current density $J(F)$. Second, the launch velocities $v_{o}$ down the side of the emitter shall also increase, but estimating their contribution requires knowledge of the behavior of the equipotential lines.

A third increase which the hemispherical boss on cylinder does not capture (at present) is a consequence

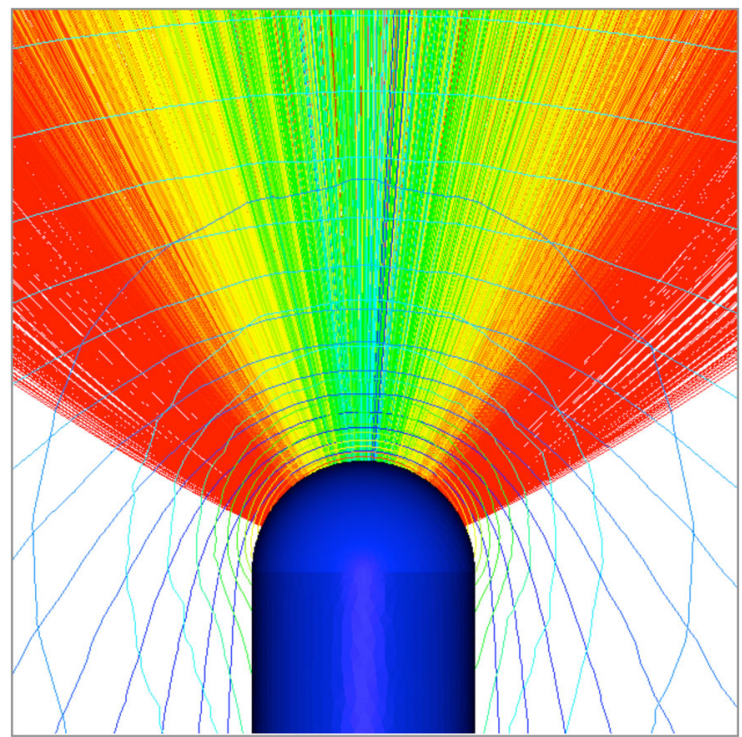

FIG. 17. Same as Fig. 3 but now the hemispherical boss sits atop a cylinder of the same length as the fibers shown in Fig. 1 (only the apex region is shown). The total current is 4 times larger and the emittance has increased by a factor of $>30$ times.

of the flatness of the apex. A close view of the trajectories and the contour lines for that case are shown in Fig. 19. As seen, the equipotential lines nevertheless show a behavior analogous to that identified in the hemispherical boss on cylinder model, and so an analytical model that reproduces those contour lines may be used in conjunction with the impulse approximation to allow a contour representing the virtual cathode surface to serve as the launch site in the PIC simulation, thereby alleviating mesh effects associated with fine gridding near the surface of the emitter and the

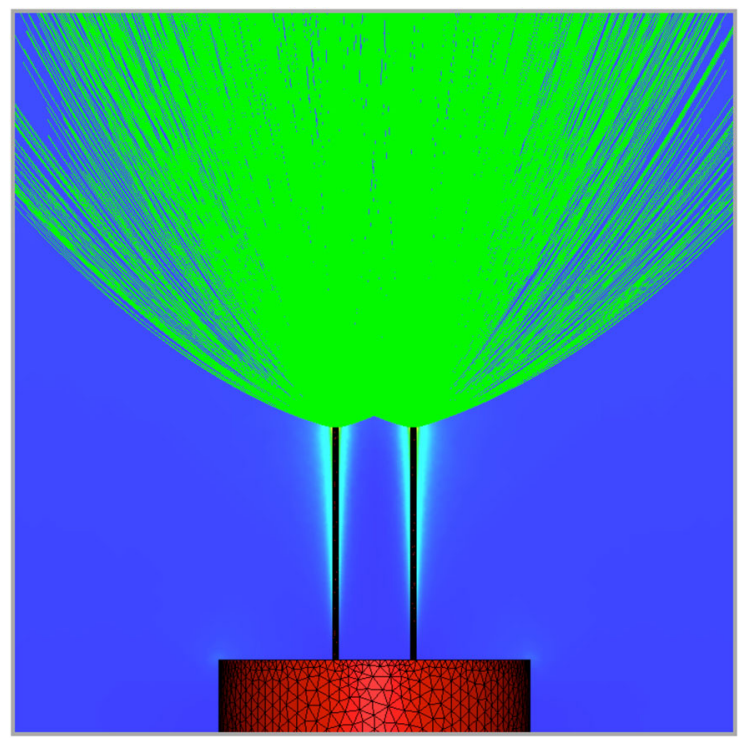

FIG. 18. PIC simulation of carbon fiber wires shown in Fig. 1. Space charge forces are included in the simulation. 


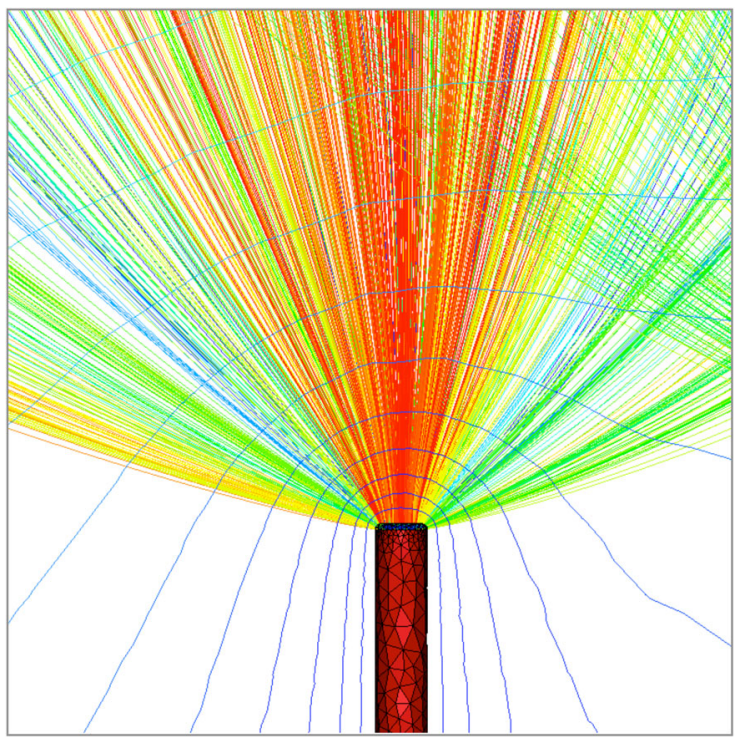

FIG. 19. Close-up of the emission region of the simulation shown in Fig. 18.

increased costs associated with finer meshes if an analytical model representing the shape is obtainable.

Such an analytical model for the wire emitters is under development and is based on a generalization of the PCM $[42,60]$ used to simulate conical (Spindt-type) field emitters, although other analytical approaches, such as prolate spheroid methods, could be used $[61,62]$, although they are in general less flexible. The PCM and its generalizations can be extended to treat an arbitrary number of emitters that may be randomly placed and exhibit variation amongst emission sites. As a result, the impact of shielding, in which adjacent

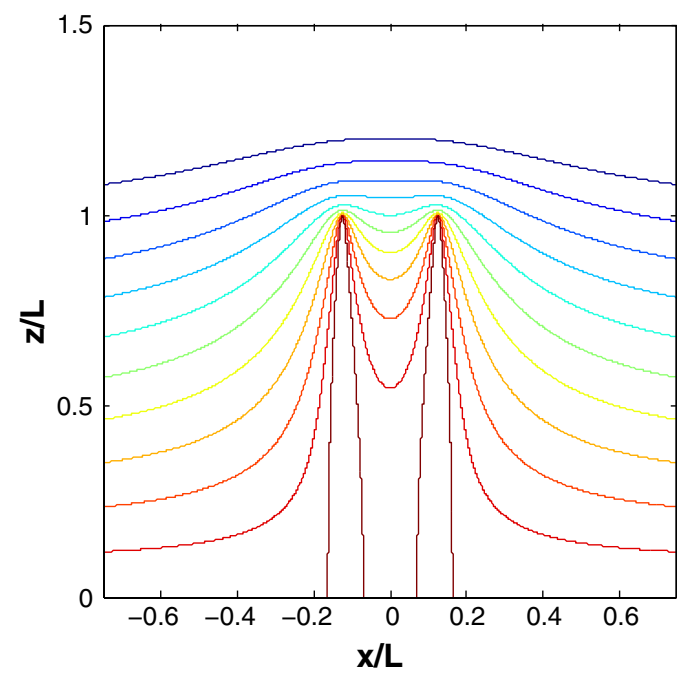

FIG. 20. Contour lines associated with an analytical model representing the carbon fibers of Fig. $1 . L$ is approximately the length of the fiber if the apex radius is small compared to the width of the fiber. The apex radius in the analytical model has been blunted to allow for easier visualization.

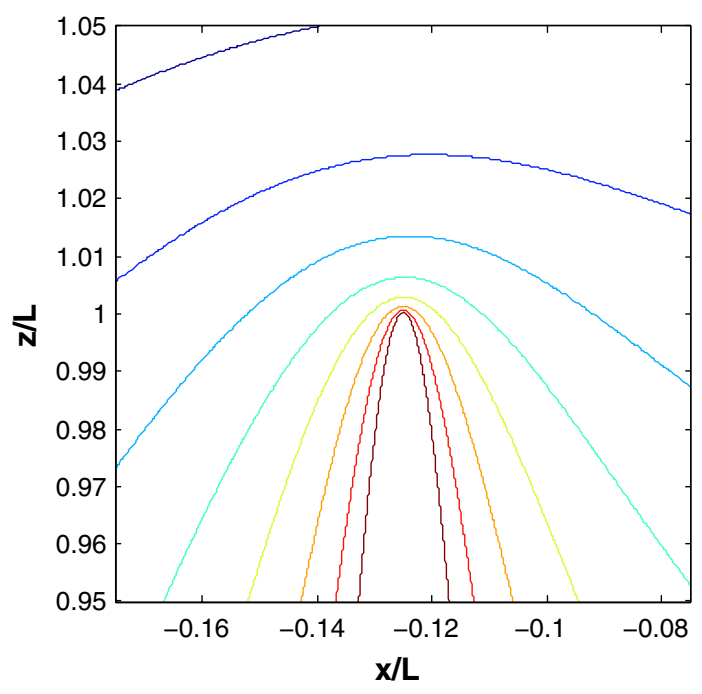

FIG. 21. Close-up of the left fiber shown in Fig. 20 and retaining the color of the contour lines shown there. The apex radius has been blunted to allow for easier visualization, but the analytic model can use any radius. Observe that the behavior matches that shown for the contour lines of Fig. 18.

close-spaced emitters can reduce the apex field on individual wirelike field emitters [63-66], can be incorporated in the model, as shown in Figs. 20 and 21. A description of the methodology and its application to random emission sites and long wires shall be given separately.

\section{DISCUSSION AND CONCLUSION}

All emission mechanisms vary with respect to surface field $F$, and therefore, the relation of the maximum current density that can be drawn across a gap to $F$ will be less than the Child-Langmuir (CL) limit given by Eq. (B1) (for which the surface field $F$ vanishes) as demonstrated in exact 1D analyses $[67,68]$. Implicit in the 1D analyses is that the field due to a sheet of charge is independent of distance from that sheet (e.g., the field due to sheet of charge with charge density $\rho d x$ is $\rho d x / 2 \varepsilon_{0}$ ). In fact, because of the axially expanding disks of charge that are emitted, the density is not uniform and instead declines as the disk area increases, and additionally, when the disk moves away from the emission site further than its diameter the field due to it begins to decline in the usual inverse-squared manner, as schematically suggested in Fig. 22.

The exact 1D space charge analysis is reasonably well approximated by a transit time model $[5,69,70]$, although there are issues if the gap is nanoscale [71]. In fact, in the case of field emission from a tungsten wire progressively covered with increasing amounts of barium to reduce the effective work function depending on coverage [72], the onset of space charge effects falls between a sequentially emitted electron model and a transit time model [34]. This suggests that the impact of space charge on the hemispherical model (and, consequently, more so for the wire 


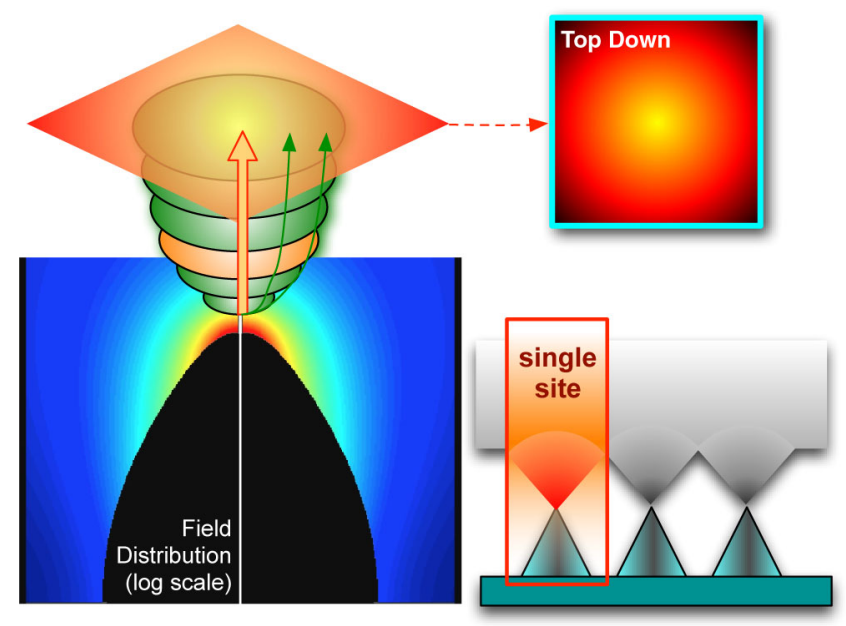

FIG. 22. Sheets of charge in the 1D formulation of space charge effects become disks of charge for emission from portrusions: the disk charge density declines as it moves away from the emission site, reducing the space charge field at the emitter apex by, first, decreasing the charge density of the disk, and second, by introducing an inverse-squared distance dependence. The example shown is for field emission from a conical tip.

models) will be twofold: first, it will depress the apex field by comparison to the off-axis fields and thereby increase the notional angle $\theta_{o}$, and second, it will augment the impulse contribution factor $\sigma$. The effect of space charge is therefore expected to increase the magnitude of $\varepsilon_{\text {array }}$ but in a decidedly nontrivial manner. An investigation of these effects is underway.

The quality of electron beams as measured by both their current and characteristic emittance is of increasing importance for high frequency vacuum electronics, high power microwave devices, and FELs, particularly when high brightness sources subject to space charge effects are used. The effects of field enhancement variation, as a consequence of the design of the cathode (as in the case of field emitters) or surface roughness (as is the case for photocathode surfaces as well as thermionic emitters) on emission characteristics and particularly emittance, is a recognized source of increased emittance that is anticipated to only be exasperated by space charge effects. In the present study, an analytic model of surface structure allows for the identification of various mechanisms that increase emittance due to roughness and emitter shape, allows for assessing the consequences of the field dependence of thermal, field, and photoemission processes, and is expected to enable estimations of the impact of space charge on emittance. The methodology is, by design, intended to be amenable to inclusion in beam optics codes to mitigate demands imposed by the small feature sizes generally associated with emission. The analytic model used in the present study is based on a hemispherical model, but the techniques developed are applicable to other analytic approaches, particularly long wires that characterize field emission from carbon fibers under investigation.
In summary, the formulation of the consequences of surface roughness (represented as an array of hemispherical protrusions) on emittance has been given. Emission from a hemispherical boss has been characterized by an emission angle, a launch velocity, and an impulse contribution factor. The separation into these factors makes possible the continued investigation of an important relation for high brightness sources, namely, the relationship between intrinsic emittance and surface field. It is argued that the method can be extended to treat nonhemispherical sources and the inclusion of space charge forces, and an indication of how that may be possible has been discussed.

\section{ACKNOWLEDGMENTS}

We gratefully acknowledge discussions and interactions with W. Tang (AFRL/RDHEC), D. W. Feldman (U. Maryland), J. Smedley (BNL), Y. Y. Lau (U. Michigan), J. Lewellen (LANL), and D. Whaley (L-3 Communications).

\section{APPENDIX A: THERMAL-FIELD- PHOTOEMISSION EQUATION}

The general thermal-field-photoemission equation $[48,60,73]$ relates the three main equations of electron emission as limiting cases after specification of the energy slope parameter for field emission $\beta_{F}\left(E_{m}\right) \equiv-\theta_{g}^{\prime}\left(E_{m}\right)$ and thermal emission $\beta_{T}=1 / k_{B} T$, followed by a reference energy $E_{o} \equiv E_{m}-\theta_{g}\left(E_{m}\right) / \theta_{g}^{\prime}\left(E_{m}\right)$ where $\theta_{g}(E)$ is the Wentzel-Kramers-Brillouin factor accounting for tunneling given by [74]

$$
\theta_{g}(E) \equiv 2 \frac{\sqrt{2 m}}{\hbar} \int_{x_{-}}^{x_{+}}\left(V\left(x^{\prime}\right)-E\right)^{1 / 2} d x^{\prime}
$$

where $x_{ \pm}(E)$ are the $E$-dependent zeros of the integrand, and where $E_{m}$ is the location of the maximum of the integrand. The ratio $n \equiv 1 /\left(k_{B} T \beta_{F}\right)$ defines the regimes of thermal and field dominated emission with $n=1$ being the boundary between them. The general current density $J$ is approximated by

$$
\begin{gathered}
J(F, T)=A_{\mathrm{RLD}} T^{2} N\left[\frac{\beta_{T}}{\beta_{F}}, \beta_{F}\left(E_{o}-\mu\right)\right], \\
N(n, s) \approx n^{2} \Sigma\left(\frac{1}{n}\right) e^{-s}+\Sigma(n) e^{-n s}, \\
N(n,-s) \approx \frac{1}{2}(n s)^{2}+\zeta(2)\left(n^{2}+1\right) .
\end{gathered}
$$

Thermal-field emission is described by Eq. (A3) and photoemission by Eq. (A4). For small argument,

$$
\Sigma(x) \approx \frac{1+x^{2}}{1-x^{2}}+\left(\frac{\pi^{2}}{6}-2\right) x^{2}+\left(\frac{7 \pi^{4}}{360}-2\right) x^{4}
$$


so that $\Sigma(x)$ provides thermal corrections to field emission and field corrections to thermal emission.

\section{Richardson-Laue-Dushman (thermal)}

Thermal emission dominates when $n<1$ for which $J(n \ll 1) \rightarrow J_{\mathrm{RLD}}$ becomes equivalent to the RichardsonLaue-Dushman equation [75]

$$
J_{\mathrm{RLD}}(F, T)=A_{\mathrm{RLD}} T^{2} \exp \left(-\frac{\phi}{k_{B} T}\right),
$$

where $\phi=\Phi-\sqrt{4 Q F}$ accounts for Schottky barrier lowering due to the field $F$ at the surface and the other terms are as in Table II.

\section{Fowler Nordheim (field)}

Field emission dominates when $n>1$ for which $J(n \gg$ 1) $\rightarrow J_{\mathrm{FN}}$ becomes equivalent to the Murphy and Good formulation of the Fowler Nordheim equation [76]. If the Deane and Forbes [77,78] approximation is used, then for the elliptical integral functions

$$
\begin{aligned}
& v(y)=1-\frac{1}{3} y^{2}[3-\ln (y)], \\
& t(y)=1+\frac{1}{9} y^{2}[1-\ln (y)] .
\end{aligned}
$$

As $t(y)$ is slowly varying with field it may be approximated by a constant: a convenient choice [74] is requiring $\partial_{x} v(\sqrt{x})=-1$ for $\sqrt{x}=y \rightarrow y_{o}$, which sets $y_{o}=e^{-1 / 2}$ and $t_{o} \equiv t\left(y_{o}\right)=1+(1 / 6 e)=1.0613$ [if extended to $v(y)$, that choice would lead to the approximation $v(y) \approx 1-(1 / 6 e)-y^{2}=0.9387-y^{2}$, similar to the Spindt approximation [79] $v(y) \approx 0.95-y^{2}$, but such parabolic approximations for $v(y)$ are not used here]. Using Table II it follows that

$$
\begin{aligned}
J_{\mathrm{FN}}(F) \rightarrow \tilde{A} F^{2-\nu} \exp \left(-B \frac{\Phi^{3 / 2}}{F}\right), \\
\nu \equiv \frac{8 Q}{9 \hbar} \sqrt{\frac{2 m}{\Phi}} \\
\tilde{A} \equiv \frac{A}{\Phi t_{o}^{2}}\left(\frac{\Phi^{2} e^{6}}{4 Q}\right)^{\nu} .
\end{aligned}
$$

\section{Fowler Dubridge (photo)}

Photoemission dominates when the electron energy in the transmission probability is augmented by the photon energy $\hbar \omega$ and the use of $N(n,-s)$ is required. Quantum efficiency $Q E$ is proportional to the ratio of the current density with the incident laser intensity. The FowlerDubridge equation is the small $n$ limit $[80,81]$

$$
Q E_{\mathrm{FD}} \propto(\hbar \omega-\phi)^{2}+2 \zeta(2)\left(k_{B} T\right)^{2} .
$$

\section{APPENDIX B: CHILD LANGMUIR (SPACE CHARGE)}

In a diode configuration, the maximum current that can be transported across an anode-cathode (AK) gap $D$ for which the anode potential is $\varphi \equiv V_{a} / q$ is governed by the Child-Langmuir relation, which assumes that the field at the surface is reduced to zero by the presence of charge in the AK region $[70,82,83]$. It reads

$$
J_{\mathrm{CL}}=\frac{4 \varepsilon_{0}}{9} \sqrt{\frac{2 q}{m}}\left(\frac{\varphi^{3 / 2}}{D^{2}}\right)=\frac{16 \pi}{9} \frac{A}{\sqrt{R_{y}}}\left(\frac{V_{a}^{3 / 2}}{D^{2}}\right),
$$

where the first more commonly encountered form is less convenient for numerical calculations herein than the second equivalent form.

\section{APPENDIX C: 1D EMITTANCE EQUATIONS}

The emittance associated with a flat thermionic cathode is well known [1]. Comparable equations for photoemission [43,47] and field emission [33] are more recent. For a flat cathode of radius $\rho_{c}$, forms here for all three are given by [after Eqs. (2), (3) and (6) of Ref. [33]]

$$
\begin{gathered}
\varepsilon_{1 D}^{T}=\frac{\rho_{c}}{2}\left(\frac{k_{B} T}{m c^{2}}\right)^{1 / 2}, \\
\varepsilon_{1 D}^{F}=\rho_{c}\left(\frac{\hbar k_{F}}{m c}\right)\left[\frac{4 \mu t_{o}}{\hbar F} \sqrt{2 m \Phi}-1\right]^{-1 / 2}, \\
\varepsilon_{1 D}^{\omega}=\frac{\rho_{c}}{2}\left(\frac{\hbar \omega-\phi}{3 m c^{2}}\right)^{1 / 2},
\end{gathered}
$$

where the superscripts " $T$," " $F$," and " $\omega$ " denote thermal, field, and photoemission, respectively.

[1] M. Reiser, Theory and Design of Charged Particle Beams, Wiley Series in Beam Physics and Accelerator Technology (Wiley, New York, 1994), p. xix.

[2] J. Booske, Phys. Plasmas 15, 055502 (2008).

[3] J. X. Qiu, B. Levush, J. Pasour, A. Katz, C. M. Armstrong, D. R. Whaley, J. Tucek, K. Kreischer, and D. Gallagher, IEEE Microw. Mag. 10, 38 (2009).

[4] D. Whaley, B. Gannon, C. Smith, C. Armstrong, and C. Spindt, IEEE Trans. Plasma Sci. 28, 727 (2000).

[5] A. Pedersen, A. Manolescu, and A. Valfells, Phys. Rev. Lett. 104, 175002 (2010).

[6] D. Shiffler, M. Ruebush, D. Zagar, M. LaCour, K. Golby, M. Clark, M. Haworth, and R. Umstattd, IEEE Trans. Plasma Sci. 30, 1592 (2002). 
[7] M. Jones, V. Neculaes, R. Gilgenbach, W. White, M. Lopez, Y. Lau, T. Spencer, and D. Price, Rev. Sci. Instrum. 75, 2976 (2004).

[8] D. Shiffler, J. Luginsland, M. Ruebush, M. Lacour, K. Golby, K. Cartwright, M. Haworth, and T. Spencer, IEEE Trans. Plasma Sci. 32, 1262 (2004).

[9] P. G. O'Shea, S. C. Bender, D. A. Byrd, J. W. Early, D. W. Feldman, C. M. Fortgang, J. C. Goldstein, B. E. Newnam, R. L. Sheffield, R. W. Warren, and T. J. Zaugg, Phys. Rev. Lett. 71, 3661 (1993).

[10] J. Feldhaus, J. Arthur, and J. Hastings, J. Phys. B 38, S799 (2005).

[11] S. Leemann, A. Streun, and A. Wrulich, Phys. Rev. ST Accel. Beams 10, 071302 (2007).

[12] C. Lee, Appl. Phys. Lett. 44, 565 (1984).

[13] T. Mayer, D. Adams, and B. Marder, J. Vac. Sci. Technol. B 14, 2438 (1996).

[14] J. Lewellen, in Proceedings of the 21st Particle Accelerator Conference, Knoxville, TN, 2005 (IEEE, Piscataway, NJ, 2005), pp. 563-567.

[15] J. H. Booske, R. J. Dobbs, C. D. Joye, C. L. Kory, G. R. Neil, G.-S. Park, J. Park, and R. J. Temkin, IEEE Trans. Terahertz Sci. Technol. 1, 54 (2011).

[16] D. Whaley, R. Duggal, C. Armstrong, C. Bellew, C. Holland, and C. Spindt, IEEE Trans. Electron Devices 56, 896 (2009).

[17] R. Longo, J. Appl. Phys. 94, 6966 (2003).

[18] D. R. Whaley, IEEE Trans. Electron Devices (to be published).

[19] L. Serafini and J. B. Rosenzweig, Phys. Rev. E 55, 7565 (1997).

[20] The Alfven-Lawson current $I_{o}$ is more commonly represented as $4 \pi \varepsilon_{0} m c^{3} / q$ in MKSA units-the equivalent form used herein is a consequence of using $\alpha$.

[21] Y. Ding, A. Brachmann, F. J. Decker, D. Dowell, P. Emma, J. Frisch, S. Gilevich, G. Hays, P. Hering, Z. Huang, R. Iverson, H. Loos, A. Miahnahri, H. D. Nuhn, D. Ratner, J. Turner, J. Welch, W. White, and J. Wu, Phys. Rev. Lett. 102254801 (2009).

[22] D. Dowell, I. Bazarov, B. Dunham, K. Harkay, C. Hernandez-Garcia, R. Legg, H. Padmore, T. Rao, J. Smedley, and W. Wan, Nucl. Instrum. Methods Phys. Res., Sect. A 622, 685 (2010).

[23] G. R. Neil and L. Merminga, Rev. Mod. Phys. 74, 685 (2002).

[24] Scientific Assessment of High-power Free-electron Laser Technology Committee on a Scientific Assessment of Free-Electron Laser Technology for Naval Applications, National Research Council (National Academies Press, Washington, DC, 2009), p. 66.

[25] C. Pellegrini and S. Reiche, IEEE J. Selected Topics Quantum Electronics 10, 1393 (2004).

[26] J. Petillo, C. Kostas, D. Panagos, S. Ovtchinnikov, A. Burke, T. Antonsen, E. Wright, K. Nguyen, E. Nelson, B. Held, J. DeFord, K. Jensen, J. Pasour, B. Levush, and L. Ludeking, in Proceedings of the IEEE International Vacuum Electronics Conference/International Vacuum Electron Sources Conference (IVEC/IVESC) 2012 (IEEE, Monterey, CA, 2012), pp. 341-342.
[27] T. Vecchione, I. Ben-Zvi, D. H. Dowell, J. Feng, T. Rao, J. Smedley, W. Wan, and H. A. Padmore, Appl. Phys. Lett. 99, 034103 (2011).

[28] D. Bradley, M. Allenson, and B. Holeman, J. Phys. D 10, 111 (1977).

[29] J. Smedley, T. Rao, and Q. Zhao, J. Appl. Phys. 98, 043111 (2005).

[30] J. Smedley, T. Rao, J. Sekutowicz, P. Kneisel, J. Langner, P. Strzyzewski, R. Lefferts, and A. Lipski, in Proceedings of the 21 st Particle Accelerator Conference, Knoxville, TN, 2005 (IEEE, Piscataway, NJ, 2005), pp. 4099-4101.

[31] S. Karkare and I. Bazarov, Appl. Phys. Lett. 98, 094104 (2011).

[32] H. J. Qian, C. Li, Y. C. Du, L. X. Yan, J. F. Hua, W. H. Huang, and C. X. Tang, Phys. Rev. ST Accel. Beams 15, 040102 (2012).

[33] K. L. Jensen, P. G. O'Shea, D. W. Feldman, and J. L. Shaw, J. Appl. Phys. 107, 014903 (2010).

[34] K. L. Jensen, J. Appl. Phys. 107, 014905 (2010).

[35] J. J. Petillo, E. Nelson, J. Deford, N. Dionne, and B. Levush, IEEE Trans. Electron Devices 52, 742 (2005).

[36] R. U. Martinelli, Appl. Opt. 12, 1841 (1973).

[37] W. Graves, L. DiMauro, R. Heese, E. Johnson, J. Rose, J. Rudati, T. Shaftan, and B. Sheehy, in Proceedings of the Particle Accelerator Conference, Chicago, IL, 2001 (IEEE, New York, 2001), p. 2227.

[38] M. Krasilnikov, Proceedings of FEL Conference (BESSY, Berlin, Germany, 2006). pp. 583-586.

[39] Y. Lau, J. Appl. Phys. 61, 36 (1987).

[40] P. Zhang, Y. Y. Lau, and R. M. Gilgenbach, J. Appl. Phys. 105, 114908 (2009).

[41] D. Dowell, H. Padmore, T. Vecchione, J. Schmerge, and W. Wan, in Photocathode Physics for Photoinjectors Workshop (Cornell University, Ithaca, NY, 2012).

[42] K. L. Jensen, J. Vac. Sci. Technol. B 29, 02B101 (2011).

[43] K. L. Jensen, P. G. O'Shea, and D. W. Feldman, Phys. Rev. ST Accel. Beams 13, 080704 (2010).

[44] C. Brau, in Physics and Applications of High Brightness Electron Beams: Proceedings of the ICFA Workshop Chia Laguna, Sardinia, Italy, 2002, edited by J. Rosenzweig, G. Travish, and L. Serafini (World Scientific, Singapore, 2004), p. 20.

[45] P. G. O’Shea, Phys. Rev. E 57, 1081 (1998).

[46] C. Lee, P. Oettinger, E. Pugh, R. Klinkowstein, J. Jacob, J. Fraser, and R. Sheffield, IEEE Trans. Nucl. Sci. 32, 3045 (1985).

[47] D. H. Dowell and J. F. Schmerge, Phys. Rev. ST Accel. Beams 12, 074201 (2009).

[48] K. L. Jensen, J. Appl. Phys. 102, 024911 (2007).

[49] R. G. Forbes and K. L. Jensen, Ultramicroscopy 89, 17 (2001).

[50] J. Lewellen (private communication).

[51] J. W. Lewellen and J. Noonan, Phys. Rev. ST Accel. Beams 8, 033502 (2005).

[52] J. R. Harris, J. W. Lewellen, and B. R. Poole, J. Appl. Phys. 112, 023304 (2012).

[53] T. Vecchione, J. Feng, W. Wan, H. A. Padmore, I. Ben-Zvi, X. Liang, M. Ruiz-Oses, T. Rao, J. Smedley, and D. Dowell, in Proceedings of the 3rd International Particle 
Accelerator Conference, New Orleans, LA, 2012 (IEEE, Piscataway, NJ, 2012), MOPPP041.

[54] R. A. Murphy and M. A. Kodis, in Vacuum Microelectronics, edited by W. Zhu (Wiley, New York, 2001), p. 349.

[55] K. L. Jensen, Y. Lau, and N. Jordan, Appl. Phys. Lett. 88, 164105 (2006).

[56] D. H. Dowell, K. J. Davis, K. D. Friddell, E. L. Tyson, C. A. Lancaster, L. D. Milliman, R. E. Rodenburg, T. Aas, M. Bemes, S. Z. Bethel, P. E. Johnson, K. Murphy, C. Whelen, J. L. Adamski, D. J. Pistoresi, D. R. Shoffstall, G. E. Busch, and D. K. Remelius, Proc. SPIE Int. Soc. Opt. Eng. 2013, 13 (1993).

[57] F. Sannibale, D. Filippetto, C. F. Papadopoulos, J. Staples, R. Wells, B. Bailey, K. Baptiste, J. Corlett, C. Cork, S. De Santis, S. Dimaggio, L. Doolittle, J. Doyle, J. Feng, D. G. Quintas, G. Huang, H. Huang, T. Kramasz, S. Kwiatkowski, R. Lellinger, V. Moroz, W. E. Norum, H. Padmore, C. Pappas, G. Portmann, T. Vecchione, M. Vinco, M. Zolotorev, and F. Zucca, Phys. Rev. ST Accel. Beams 15 (2012).

[58] J. Maldonado, P. Pianetta, D. Dowell, J. Smedley, and P. Kneisel, J. Appl. Phys. 107, 013106 (2010).

[59] J. Smedley (private communication).

[60] K. L. Jensen, Y. Y. Lau, D. W. Feldman, and P. G. O'Shea, Phys. Rev. ST Accel. Beams 11, 081001 (2008).

[61] J. Zuber, K. L. Jensen, and T. Sullivan, J. Appl. Phys. 91, 9379 (2002).

[62] K. L. Jensen, D. W. Feldman, and P. G. O'Shea, J. Vac. Sci. Technol. B 23, 621 (2005).

[63] O. Groning, O. Kuttel, C. Emmenegger, P. Groning, and L. Schlapbach, J. Vac. Sci. Technol. B 18, 665 (2000).

[64] K. Teo, E. Minoux, L. Hudanski, F. Peauger, J. Schnell, L. Gangloff, P. Legagneux, D. Dieumegard, G. Amaratunga, and W. Milne, Nature (London) 437, 968 (2005).
[65] W. Tang, D. Shiffler, and K. L. Cartwright, J. Appl. Phys. 110, 034905 (2011)

[66] W. Tang, D. Shiffler, K. Golby, M. LaCour, and T. Knowles, J. Vac. Sci. Technol. B 30, 061803 (2012).

[67] R. Forbes, J. Appl. Phys. 104, 084303 (2008).

[68] A. Rokhlenko, K. L. Jensen, and J. L. Lebowitz, J. Appl. Phys. 107, 014904 (2010).

[69] M.E. Griswold, N. J. Fisch, and J.S. Wurtele, Phys. Plasmas 17, 114503 (2010).

[70] K. L. Jensen, J. Lebowitz, Y. Y. Lau, and J. Luginsland, J. Appl. Phys. 111, 054917 (2012).

[71] L. K. Ang, W. S. Koh, Y. Y. Lau, and T. J. T. Kwan, Phys. Plasmas 13, 056701 (2006).

[72] J. P. Barbour, W. Dolan, J. Trolan, E. Martin, and W. Dyke, Phys. Rev. 92, 45 (1953).

[73] K. L. Jensen, Advances in Imaging and Electron Physics, Electron Emission Physics, edited by P. Hawkes (Academic Press, San Diego, CA, 2007), Vol. 149.

[74] K. L. Jensen, J. Appl. Phys. 111, 054916 (2012).

[75] C. Herring and M. Nichols, Rev. Mod. Phys. 21, 185 (1949).

[76] E. L. Murphy and R. H. Good, Phys. Rev. 102, 1464 (1956).

[77] R. G. Forbes, Appl. Phys. Lett. 89, 113122 (2006).

[78] J. H. B. Deane and R. G. Forbes, J. Phys. A 41, 395301 (2008).

[79] C. A. Spindt, I. Brodie, L. Humphrey, and E. R. Westerberg, J. Appl. Phys. 47, 5248 (1976).

[80] L. A. Dubridge, Phys. Rev. 43, 727 (1933).

[81] K. L. Jensen, N. A. Moody, D. W. Feldman, E. J. Montgomery, and P. G. O'Shea, J. Appl. Phys. 102, 074902 (2007).

[82] I. Langmuir, Phys. Rev. 2, 450 (1913).

[83] J. Luginsland, Y. Lau, R. Umstattd, and J. Watrous, Phys. Plasmas 9, 2371 (2002). 